\title{
High Persistence and Nonlinear Behavior in Financial Variables: A More Powerful Unit Root Testing in the ESTAR Framework
}

\author{
Tolga Omay ${ }^{1}$, Aysegul Corakci ${ }^{2}$ and Esra Hasdemir ${ }^{3, *}$ \\ 1 Department of Economics, Atilim University, 06830 Ankara, Turkey; tolga.omay@atilim.edu.tr \\ 2 Department of Economics, Çankaya University, 06790 Ankara, Turkey; aeruygur@cankaya.edu.tr \\ 3 Department of Logistics Management, University of Turkish Aeronautical Association, 06790 Ankara, Turkey \\ * Correspondence: ehasdemir@thk.edu.tr
}

Citation: Omay, T.; Corakci, A.; Hasdemir, E. High Persistence and Nonlinear Behavior in Financial Variables: A More Powerful Unit Root Testing in the ESTAR Framework. Mathematics 2021, 9, 2534. https:// doi.org/10.3390/math9202534

Academic Editor: Bahram Adrangi

Received: 31 July 2021

Accepted: 1 October 2021

Published: 9 October 2021

Publisher's Note: MDPI stays neutral with regard to jurisdictional claims in published maps and institutional affiliations.

Copyright: (c) 2021 by the authors. Licensee MDPI, Basel, Switzerland. This article is an open access article distributed under the terms and conditions of the Creative Commons Attribution (CC BY) license (https:/ / creativecommons.org/licenses/by/ $4.0 /)$.

\begin{abstract}
In this study, we consider the hybrid nonlinear features of the Exponential Smooth Transition Autoregressive-Fractional Fourier Function (ESTAR-FFF) form unit root test. As is well known, when developing a unit root test for the ESTAR model, linearization is performed by the Taylor approximation, and thereby the nuisance parameter problem is eliminated. Although this linearization process leads to a certain amount of information loss in the unit root testing equation, it also causes the resulting test to be more accessible and consistent. The method that we propose here contributes to the literature in three important ways. First, it reduces the information loss that arises due to the Taylor expansion. Second, the research to date has tended to misinterpret the Fourier function used with the Kapetanios, Shin and Snell (2003) (KSS) unit root test and considers it to capture multiple smooth transition structural breaks. The simulation studies that we carry out in this study clearly show that the Fourier function only restores the Taylor residuals of the ESTAR type function rather than accounting forthe smooth structural break. Third, the new nonlinear unit root test developed in this paper has very strong power in the highly persistent near unit root environment that the financial data exhibit. The application of the Kapetanios Shin Snell- Fractional Fourier (KSS-FF) test to ex-post real interest rates data of 11 OECD countries for country-specific sample periods shows that the new test catches nonlinear stationarity in many more countries than the KSS test itself.
\end{abstract}

Keywords: ESTAR; unit root; fractional frequency fourier function; near unit root; high persistency; nonlinear financial variables

\section{Introduction}

Stochastic properties of financial variables are examined in many studies. Existing research has established that financial variables exhibit state-dependent nonlinearity and high persistence. Economic theory proposes several justifications for nonlinear behavior in financial series. The presence of market frictions and transaction costs are two of the most frequently cited factors contributing to nonlinear adjustment in the literature. The spread between financial prices, short sale phenomenon and borrowing restrictions and other transaction costs makes arbitrage unprofitable for small deviations of asset prices or returns from their equilibrium values. Therefore, a return to equilibrium only occurs when the deviations from this equilibrium are large, and thus arbitrage activities are profitable [1]. That is to say, the dynamic behavior of returns will differ according to the size of the deviation from equilibrium regardless of the sign of this deviation, leading to asymmetric dynamics for returns of different sizes [2-5]. In addition to market frictions and transaction costs, the interaction among heterogeneous agents $[4,6]$ and the diversity of trustees' beliefs [7,8] may also cause persistent deviations from asset price equilibrium. On the other hand, heterogeneity in investors' goals resulting from changing investment horizons and risk profiles [9], herd behavior or momentum trading [10] may reveal different dynamics depending on the market behavior. 
Analysis of nonlinear behavior in financial markets was first carried out by Kapetanios, Shin and Snell (2003) [11] (hereafter, KSS). In their seminal study, KSS developed a unit root test statistic for financial data exhibiting nonlinear stochastic properties and concluded that financial data would be nonlinear stationary in the near unit root case (In the recent literature, there are a vast number of studies that analyze bivariate or multivariate nonlinearity in a time series or in a panel setting besides the studies that focus on univariate time series nonlinearity (see; Omay and Kan (2010) [12]; Eryugur and Omay (2014) [13]; Batrancea et al., (2019) [14])). The nonlinear transition function used in the KSS test is an ESTAR (1) type of function that captures all the nonlinear economic features of financial data mentioned above. However, the KSS unit root testing procedure is based on examining the unit root in the transition speed parameter, which cannot be tested directly due to the nuisance parameter problem. To circumvent this, KSS compute the first-order Taylor expansion of the ESTAR (1) function and obtain an auxiliary regression to test for the unit root null. Although this approach provides a simple solution to test for nonlinearity in the near unit root case, applying the Taylor expansion may also cause the power of the test to decrease. The main aim of this study is to introduce an alternative method to increase the power of the ESTAR type unit root test, which is based on using the fractional Fourier function.

In the literature, there are a few studies that suggest using the Fourier function with an ESTAR type unit root test. Christopoulos and León-Ledesma (2010) [15] (hereafter, FKSS) have proposed a unit root test by incorporating the integer frequency Fourier function into the KSS test. According to the findings of [15], there are multiple smooth transition integer frequency Fourier trends in the real exchange rate series and there is an ESTAR (1) type convergence to this smooth transition trend. In another study Emirmahmutoglu, Omay, Shahzad and Mohd Nor (2021) [16] have studied the asymptotic properties of the FKSS test developed by [15] and augmented their power analysis with other simulations. The study in [16] has detected anomalies in the power findings of [15] and has shown that the power of the test developed by Enders and Lee $(2012 a, b)([17,18])$ (hereafter, FADF) was higher than the data-generating process (DGP) of FKSS. Moreover, Ref. [15] pointed out that the amplitudes of the sine and cosine variables used in the power analysis were given symmetrically. Ref. [16], by using these symmetric amplitude parameters in their study, have reached similar results. Therefore, Ref. [16] criticized [15] on the over filtration problem of the integer frequency Fourier function. This over filtration problem may, in turn, cause misinterpretations about structural breaks and state-dependent nonlinearity. We focus on showing the interpretation of the test rather than the over filtration problem in this study.

As mentioned above, computing the Taylor expansion for approximating the ESTAR (1) function causes information loss in the ESTAR function. This information loss may be stored in the Taylor residuals which are considered to be a modeling error or an omitted functional form. The simplest way to restore Taylor residuals is utilizing the Fourier functions that converge to any function of an unknown structure with the Sobolev norm [16]. In the light of this property of Fourier functions, it can be said that it would be misleading to see the Fourier function just as a function that catches smooth transitional multiple breaks as in $[15,17,18]$. Our simulation study in Section 2 shows that the Fourier function can approximate Taylor residuals. Besides, in the power analysis, we demonstrate that some important information about the nonlinear structure, which was lost by the Taylor expansion, is restored using the Fourier function.

Ref. [15] utilizes the integer frequency Fourier function to test the structural break and state-dependent nonlinearity. In this study, we use the fractional frequency Fourier function, following Omay (2015) (henceforth, FFFFF) [19]. Ref. [19] concludes that model misspecification disappears if fractional frequency Fourier function is used instead of the integer frequency Fourier function. Therefore, another contribution of this study to the literature is obtaining the asymptotic distributions of the newly proposed test that prevents model misspecification. In other words, we have developed a more efficient ESTAR type 
state-dependent nonlinear unit root test by combining the KSS test developed by [11,19] test (hereafter, KSS-FF test). The KSS-FF test that we propose in this study can account for state-dependent nonlinearity and high persistence properties of financial data much better.

The organization of this study is as follows: In Section 2, the theoretical background, the derivation, critical values, asymptotic distribution and the small sample properties of the test are provided. In Section 3, the limitations of the test are discussed. In Section 4, the proposed test is applied to the ex-post real interest rates of 11 OECD countries, and Section 5 is devoted to the concluding remarks.

\section{Theoretical Background}

\subsection{Approximating the Taylor Residuals via the Fourier Function for the ESTAR (1) Unit Root} Test

This section will show that the Fourier function can be used to increase the power of an ESTAR type unit root test obtained by computing the Taylor expansion. Ref. [11] mentioned the importance of the transition speed parameter while producing the ESTAR type unit root test. When the transition speed parameter is zero, there is no nonlinearity, and the unit root problem arises. In order to understand the situation better, it is helpful to summarize the study of [11].

$$
y_{t}=\beta y_{t-1}+\theta y_{t-1} G\left(\gamma ; y_{t-1}\right)+\widetilde{\varepsilon}_{t}
$$

where $\varepsilon_{t} \sim \operatorname{iid}\left(0, \sigma^{2}\right), \beta$ and $\theta$ are unknown parameters. $y_{t}$ is a stochastic process with zero-mean. Ref. [11] uses the ESTAR function as a transition function.

$$
G\left(\gamma ; y_{t-d}\right)=1-\exp \left(-\gamma y_{t-d}^{2}\right)
$$

In Equation (2), $y_{t}$ is the transition variable where $\gamma>0$ and $d>1$ denote the speed of the mean reversion and the lag of the transition variable, respectively. Moreover, $y_{t}$ is a bounded function between 0 and 1 .

We can define the model by inserting Equation (2) into Equation (1):

$$
y_{t}=\beta y_{t-1}+\theta y_{t-1}\left(1-\exp \left(-\gamma y_{t-d}^{2}\right)\right)+\widetilde{\varepsilon}_{t}
$$

When we rewrite Equation (3) in terms of the traditional unit root testing methodology, we obtain Equation (4) as follows:

$$
\Delta y_{t}=\phi y_{t-1}+\theta y_{t-1}\left(1-\exp \left(-\gamma y_{t-d}^{2}\right)\right)+\varepsilon_{t}
$$

where $\phi=\beta-1$ and $\gamma>0$.

In Equation (3), if $y_{t}$ is away from the attractor, it can converge to the attractor (i.e., mean of the nonlinear process). On the one hand, if $y_{t}$ is sufficiently away from the attractor, it can converge to the mean of the nonlinear system. On the other hand, if $y_{t}$ is not sufficiently away from the attractor, it exhibits some instabilities in the neighborhood of the attractor. Such behavior is also prevalent in the asset markets. If the difference between the risk-adjusted returns of two assets is large, then the profitability of arbitrage is higher than the case where the mentioned difference is small due to the transaction costs. Consequently, the speed of the mean reversion to the equilibrium changes depending on the size of this differential inversely proportionally. The second empirical application carried out by [11] was motivated by Sercu et al., (1995) [20] and Michael et al. (1997) [21], which examine the nonlinearity included in the purchasing power parity (PPP) hypothesis. These studies point out that, the larger the deviation from the PPP, the faster the mean-reverting behavior to the equilibrium. Again, the speed of the mean reversion is determined by the distance to the attractor. Here, in the framework of Equation (4), $\gamma>0$; that is, the outer regimes are convergent $\theta<0$ and inner regime is divergent $(\phi \geq 0)$, finally $\phi+\theta<0$. It states that the proposed nonlinear stochastic process is locally unit root (explosive) globally stationary. 
In these circumstances, the transition variable stays in the middle regime for values of $y_{t-1}^{2}<2$ whereas the transition variable proceeds to the outer regime when it has bigger values. Therefore, it has stable dynamics and so, it is geometrically ergodic. Ref. [11] shows that these conditions are satisfied. This nonlinear behavior can also be seen in Figure 1. In the null hypothesis, which indicates the unit root case, $\theta=0$ and $\gamma=0$ whereas in the alternative hypothesis, $\theta=0$ and $\gamma>0$. In this case, $0<\theta<-2$. So, the nonlinear ESTAR stationarity is satisfied when $\theta=0$ and $0<\theta<-2$.

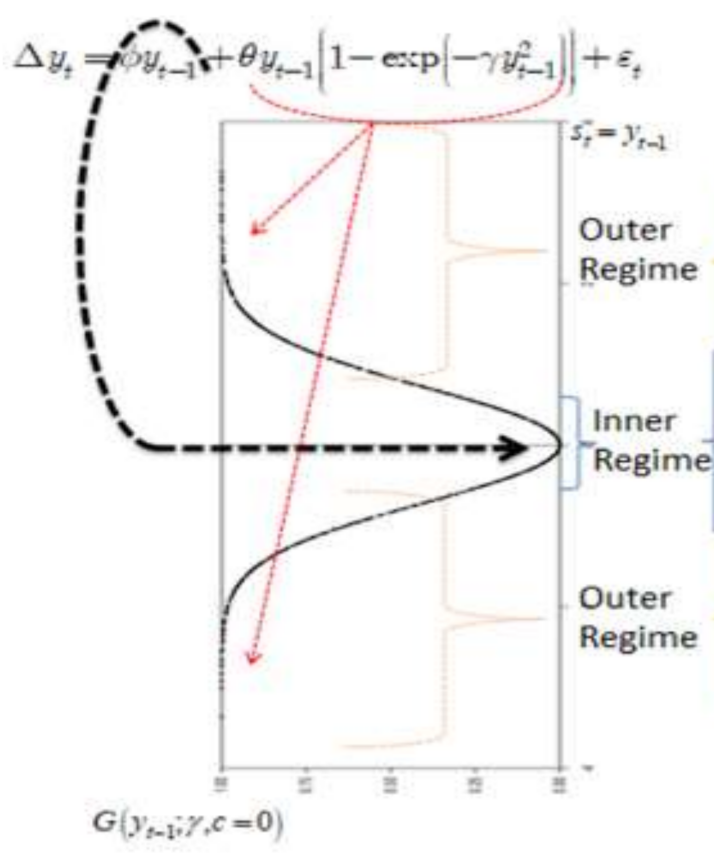

ESTAR(1) Model

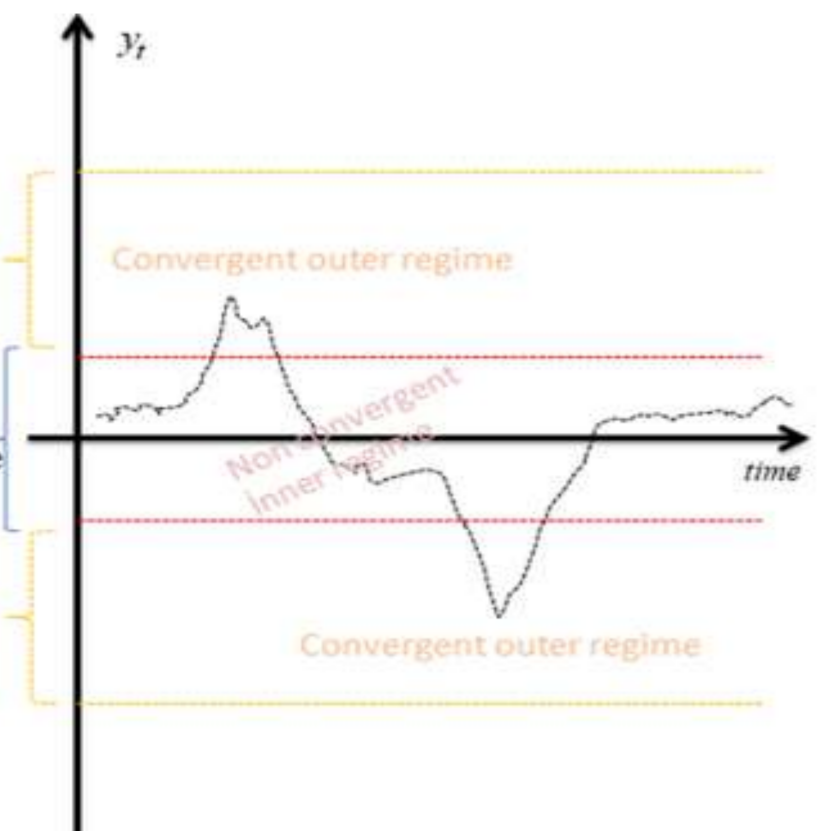

Band TAR ModeI

Figure 1. Explanation of the ESTAR (1) process using the band Threshold Autoregressive (TAR) model.

In view of these circumstances, $\phi=0$ and we follow [11] by considering the case where $d=1$. Then the model can be expressed in the following form:

$$
\Delta y_{t}=\theta y_{t-1}\left(1-\exp \left(-\gamma y_{t-1}^{2}\right)\right)+\varepsilon_{t}
$$

Since $\theta=0$, we focus on the value of $g$ to test the unit root. Thus, the null hypothesis is as follows:

$$
H_{0}: \gamma=0 \text { (Linear unit root process) }
$$

and the alternative hypothesis is:

$$
H_{1}: \gamma>0 \text { (Nonlinear stationary process) }
$$

We cannot directly test the unit root hypotheses because there is no $\gamma$ under the null hypothesis. Therefore, following Davies (1987) [22] and Luukkonen, Saikkonen and Teräsvirta (1988) [23], we apply the Taylor expansion to $\gamma=0$ under the null hypothesis.

$$
T_{1}\left(y_{t-d} ; \gamma, c=0\right)=G\left(y_{t-d} ; \gamma=0\right)+\left.\gamma \frac{G\left(y_{t-d} ; \gamma=0\right)}{\partial \gamma}\right|_{\gamma=0}+R_{1}\left(y_{t-d} ; \gamma, c=0\right) T_{1}\left(y_{t-d} ; \gamma\right)=\gamma\left(y_{t-d}\right)^{2}+R_{1}\left(y_{t-d} ; \gamma\right)
$$

We obtain the auxiliary regression as in [11] when we take $d=1$.

$$
y_{t}=\beta_{1} y_{t-1}+\beta_{2} y_{t-1}^{2}+e_{t}
$$




$$
e_{t}=\varepsilon_{t}+(\phi-\theta) y_{t-1} R_{1}\left(y_{t-1} ; \gamma\right)
$$

After some algebraic operations, we obtain the following auxiliary regression:

$$
\begin{gathered}
\Delta y_{t}=\delta y_{t-1}^{3}+\underbrace{\text { Taylor Expansion Residual + Error Term }}_{\mathrm{u}_{\mathrm{t}}} \\
\Delta y_{t}=\delta y_{t-1}^{3}+u_{t}
\end{gathered}
$$

where the null hypothesis is,

$$
H_{0}: \delta=0 \text { (Linear unit root process) }
$$

and the alternative hypothesis is

$$
H_{1}: \delta<0 \text { (Nonlinear stationary process) }
$$

Then, the $t$-type test statistics can be calculated as follows:

$$
t_{N L}=\delta / \text { s.e. }(\hat{\delta})
$$

In this study, we use the approximation of the nonlinear process instead of its actual values, where there is an information loss. The method that compensates the information loss between the approximated and actual nonlinear processes can provide testing procedures as effective as the procedures using the actual nonlinear process. This issue has already been pointed out in the literature, yet it is misinterpreted. To show the method proposed in this study, we prefer to show the residual structure of Taylor expansion.

$$
u_{t}=e_{t}+R_{t}
$$

In the above given equation, $\mathrm{R}_{\mathrm{t}}$ represents the Taylor expansion residuals that are considered as either a modeling error or an omitted functional form (such as omitted variable bias or wrong functional form) in the literature. The simplest way to restore this $R_{t}$ is with the help of Fourier functions. Fourier functions can converge to functions of unknown structures with ([16]). The Fourier function along with the ESTAR test was first used in [15] who consider the Fourier function as a smooth transition of multiple structural breaks in the FKSS test, as it is done in $[17,18]$. However, using simulation studies, [16] show that the power of the integer Fourier function-ESTAR test is smaller than the integer Fourier function test. Following [16], in this study, we will show that the use of fractional Fourier instead of integer one can improve this convergence.

$$
\mathrm{R}_{\mathbf{t}}=\varphi(t)=\alpha_{0}+\alpha_{1} \sin \left(\frac{2 \pi k^{f r} t}{T}\right)+\alpha_{2} \cos \left(\frac{2 \pi k^{f r} t}{T}\right)
$$

If the Taylor expansion can be performed as in the method given in Equation (15), the KSS-FF test proposed in this study will be considered as a state-dependent nonlinear unit root test, rather than being a test combining state-dependent nonlinearity with a structural break.

$$
\Delta y_{t}=\delta y_{t-1}^{3}+\alpha_{0}+\alpha_{1} \sin \left(\frac{2 \pi k^{f r} t}{T}\right)+\alpha_{2} \cos \left(\frac{2 \pi k^{f r} t}{T}\right)+\varepsilon_{t}
$$

Therefore,

$$
\begin{aligned}
& \theta y_{t-1}\left(1-\exp \left(-\gamma y_{t-1}^{2}\right)\right)+\varepsilon_{t}=\delta y_{t-1}^{3}+\alpha_{0}+\alpha_{1} \sin \left(\frac{2 \pi k f^{f r_{t}}}{T}\right)+\alpha_{2} \cos \left(\frac{2 \pi k^{f r_{t}}}{T}\right)+\varepsilon_{t} \\
& \theta y_{t-1}\left(1-\exp \left(-\gamma y_{t-1}^{2}\right)\right)=\delta y_{t-1}^{3}+\alpha_{0}+\alpha_{1} \sin \left(\frac{2 \pi k f^{\prime} t}{T}\right)+\alpha_{2} \cos \left(\frac{2 \pi k r^{f r_{t}}}{T}\right) \\
& \theta \cong \delta
\end{aligned}
$$


To clarify this point, we run the following simulation.

Step 1. ESTAR (1) data are generated. Then, they are regressed on the $y_{t}^{3}$ series obtained with ESTAR (1) Taylor expansion.

Step 2. Use the same ESTAR (1) data in Step 1. Then, they are regressed on the $y_{t}^{3}$ series with augmenting fractional frequency Fourier function. Obtain the estimated fractional frequency.

Step 3. Residuals obtained from these two regressions are subtracted from originally obtained residuals and the differenced series thereby obtained.

Step 4 . The distance between the original series and the cumulative summation of squared differenced residuals is calculated.

Step 5. In the case where the difference between KSS-FF residuals and original model error terms is less than the difference between KSS residuals and original model error terms, Equation (12) becomes valid.

As it is displayed in Table 1 and Figure 2, the KSS-FF model reintroduces Taylor residuals into the model in a way that satisfies Equation (12). It is remarkable that the results in which we are interested are coherent with the power analysis applied to ESTAR (1) DGP by [16]. Here, the power of the KSS test is better than that of all the other tests previously used in the literature, naturally. Nonetheless, the power of the KSS-FF test is the best in almost all parameter regions, while the second-best is KSS test and the third-best is integer frequency Fourier unit root test (FADF) used in the study of [18]. Ref. [17] claimed that the KSS test is a rival to their FADF test, but they did not compare the power of the FADF test with that of the KSS test. In this sense, the FADF test is a competitor to KSS, which is also confirmed by [16]. Nevertheless, we have shown that the KSS-FF test, which is proposed in this study, really increases the power by taking the Taylor expansion term in the KSS test, according to the simulation. Another point to emphasize is that the KSS-FF test proposed is not a test for detecting structural breaks, but a state-dependent nonlinear unit root test. The KSS-FF test is one type of the ESTAR (1) test, which exhibits information loss due to the Taylor expansion, but which regains the lost information by the fractional Fourier function. Accordingly, it would be a more appropriate terminology to describe the KSS-FF test as an ESTAR test with augmented fractional frequency Fourier trend. As it is displayed in Figure 3, this can be seen more clearly by drawing the transition function including the Taylor residuals to the model with the Fourier function. For this purpose, we use the generated data and plot them with the transition functions. To do so, we take the $y_{t}^{2}$ and $y_{t}^{2}+$ Fourier trends to the $0-1$ range to bring them to the same scale.

$$
G\left(s_{t}=y_{t-1} ; \gamma, c=0\right)
$$

Table 1. Simulation analysis for approximating the Taylor residuals with the Fourier function.

\begin{tabular}{cccccc}
\hline $\boldsymbol{\gamma}$ & $\boldsymbol{\phi}$ & KSS & $\begin{array}{c}\text { KSS-FF } \\
\mathbf{m a x} \boldsymbol{k}^{f}=10.0\end{array}$ & $\begin{array}{c}\text { Mean } \\
\boldsymbol{k}^{f}\end{array}$ & $\begin{array}{c}\text { Variance } \\
\boldsymbol{k}^{f}\end{array}$ \\
\hline 0.01 & -0.1 & 12.102 & 2.636 & 0.983 & 1.110 \\
0.01 & -0.5 & 8.859 & 2.463 & 1.111 & 1.377 \\
0.01 & -0.75 & 8.032 & 2.369 & 1.148 & 1.364 \\
0.01 & -1.0 & 7.489 & 2.299 & 1.206 & 1.500 \\
0.5 & -0.1 & 4.681 & 1.960 & 1.373 & 1.553 \\
0.5 & -0.5 & 2.463 & 1.460 & 1.752 & 1.872 \\
0.5 & -0.75 & 2.049 & 1.323 & 1.924 & 2.059 \\
0.5 & -1.0 & 1.826 & 1.246 & 2.050 & 2.229 \\
1.0 & -0.1 & 3.743 & 1.779 & 1.493 & 1.658 \\
\hline
\end{tabular}


Table 1. Cont.

\begin{tabular}{cccccc}
\hline$\gamma$ & $\boldsymbol{\phi}$ & KSS & $\begin{array}{c}\text { KSS-FF } \\
\max k^{f}=10.0\end{array}$ & $\begin{array}{c}\text { Mean } \\
k^{f}\end{array}$ & $\begin{array}{c}\text { Variance } \\
\boldsymbol{k}^{f}\end{array}$ \\
\hline 1.0 & -0.5 & 1.853 & 1.246 & 2.002 & 2.168 \\
1.0 & -0.75 & 1.557 & 1.116 & 2.260 & 2.654 \\
\hline
\end{tabular}

Note: $\mathrm{T}=200$, and draw $=10,000$. The transition speed and the phi $(\phi)$ give the structure of the function. KSS indicates the squared differences from the KSS linearized version and original series whereas KSS-FF indicates the linearized version plus the Fourier function squared differences.

KSS test residuals and original error terms.

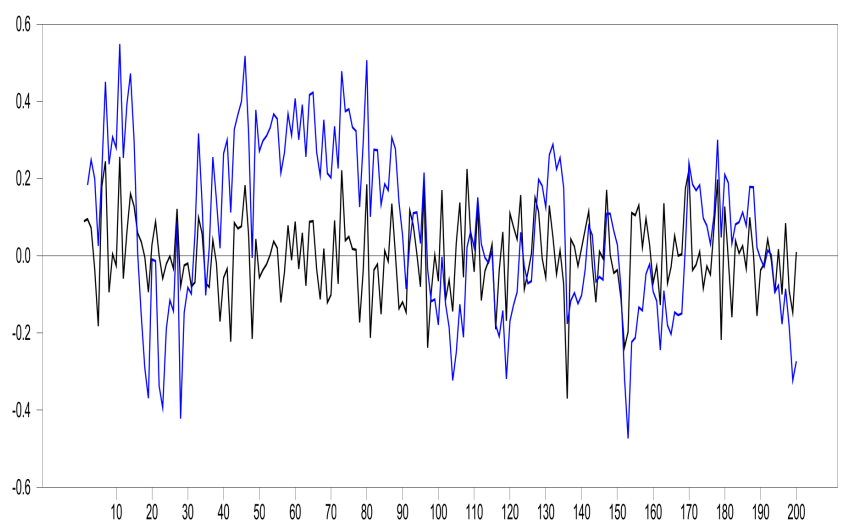

(a)

Cumulative difference: 6.513 .

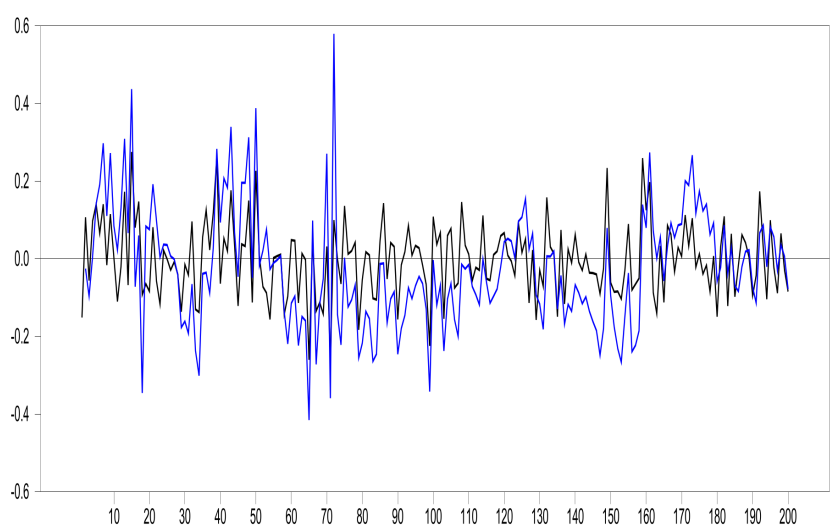

Cumulative difference: 1.787.

(c)
KSS-FF residuals and original error terms.

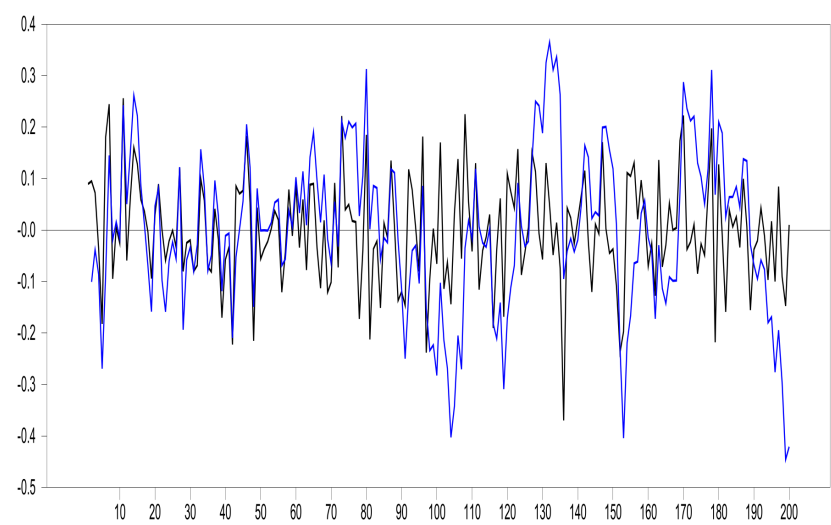

(b)

Cumulative difference: 0.899 .

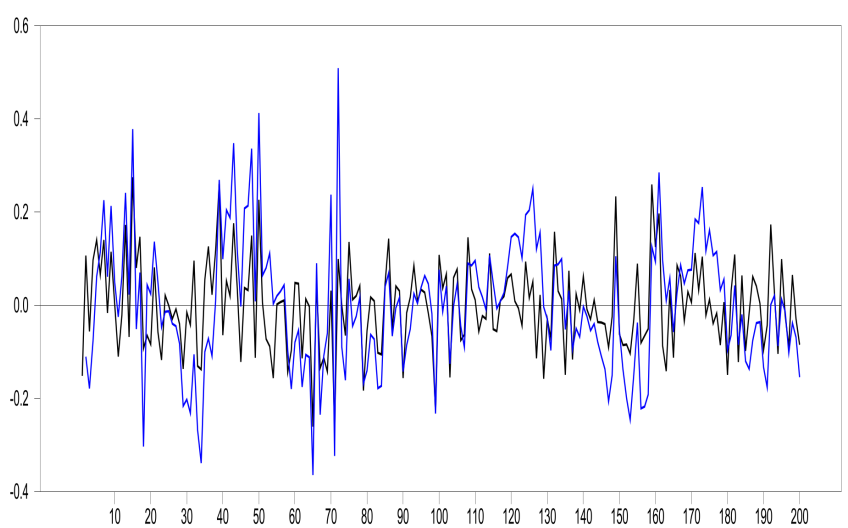

Cumulative difference: 1.316 .

(d)

Figure 2. Residuals and original error term from KSS model and ESTAR (1) fractional Fourier: The simulation results given in Table 1 are visualized in Figure 2. KSS residuals are obtained as the squared differences between the linearized version of KSS and original series whereas the KSS-FF residuals are calculated as the squared differences between the linearized version plus the Fourier function and the original series. In each panel in Figure 2, the black lines indicate the original residuals, and the blues lines indicate the estimated KSS and KSS-FF residuals. 
$\gamma=0.01 \phi=-0.1$ and $k^{f r}=1.1, \mathrm{~T}=100$

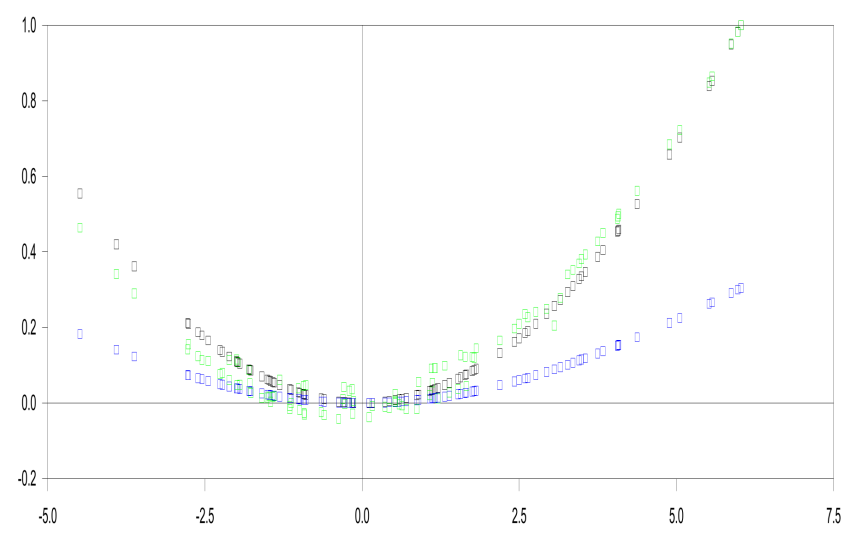

(a)

$$
\gamma=0.01 \phi=-0.75 \text { and } k^{f r}=1.2
$$

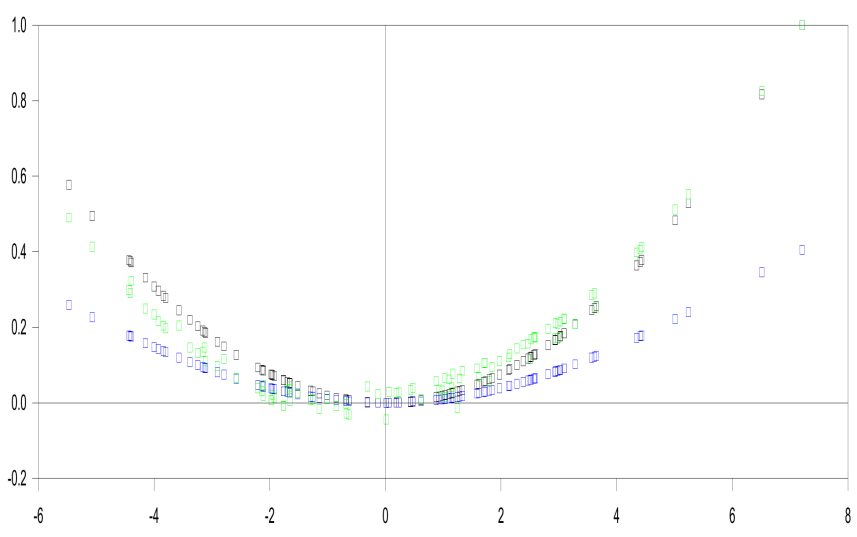

(c)

$$
\gamma=0.01 \phi=-0.5 \text { and } k^{f r}=1.5
$$

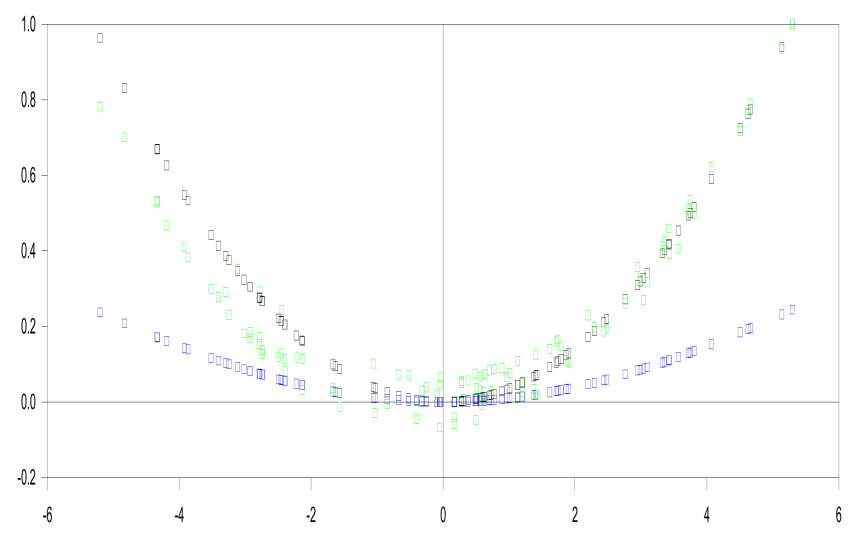

(b)

$$
\gamma=0.01 \phi=-1.0 \text { and } k^{f r}=2.2
$$

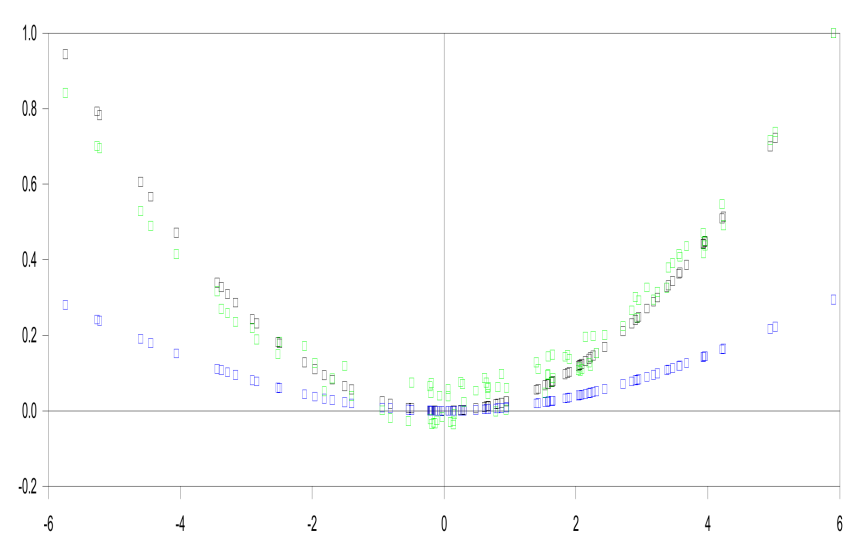

(d)

Black-generated ESTAR, green $y^{2}+$ Fourier trend, Blue $y^{2}$.

Figure 3. Generated ESTAR (1) Taylor expansion and Fourier adjustment: the parameter values are given in the boxes above each panel. The functions colored black are ESTAR functions obtained by using the parameters. The functions colored blue show the squared forms obtained by the Taylor approximation. Finally, the functions colored green display the retrieval of the original ESTAR function by adding the squared form obtained by the Taylor approximation and Fourier transformation.

\subsection{ESTAR (1) Fractional Frequency Fourier Tests and Their Asymptotic Distributions}

Let us consider the following data-generating process:

$$
\bar{\omega}_{t}=\phi \bar{\omega}_{t-1}+\theta \bar{\omega}_{t-1}\left[1-\exp \left(-\gamma \bar{\omega}_{t-d}^{2}\right)\right]+\xi_{t}
$$

where $u_{t} \sim \operatorname{iid}\left(0, \sigma^{2}\right)$ and the initial condition $\omega_{0}$ is zero. $\omega_{t}$ is a de-meaned and detrended series with fractional frequency Fourier function. Equation (18) assumes that the adjustment speed is nonlinear and follows an ESTAR process developed in [11]. In this study, we propose the two-step testing procedure of [15]. In the first step, we obtain the de-meaned or de-trended series, $\omega_{t}$ as follows:

$$
\bar{\omega}_{t}=y_{t-1}-\hat{\alpha}_{0}-\hat{\alpha}_{1} \sin \left(\frac{2 \pi \hat{k}^{f r} t}{T}\right)-\hat{\alpha}_{2} \cos \left(\frac{2 \pi \hat{k}^{f r} t}{T}\right)
$$




$$
\bar{\omega}_{t}=y_{t-1}-\hat{\alpha}_{0}-\hat{\alpha}_{1} \sin \left(\frac{2 \pi \hat{k}^{f r} t}{T}\right)-\hat{\alpha}_{1} \cos \left(\frac{2 \pi \hat{k}^{f r} t}{T}\right)-\hat{\lambda} t
$$

where $\hat{\alpha}_{0}, \hat{\alpha}_{1}, \hat{\alpha}_{2}$ and $\hat{\lambda}$ are OLS estimators for de-meaned and de-trended cases, respectively. Next, we build the ESTAR (1) augmented fractional frequency Fourier function unit root test by using the de-meaned and de-trended series $\bar{\omega}_{t}$ in the second step. Then, by applying the steps from Equation (1) until Equation (11), the following test equation is obtained:

$$
\Delta \bar{\omega}_{t}=\delta \bar{\omega}_{t-1}^{3}+\eta_{t}
$$

where $\eta_{t}=e_{t}+R_{t}$ with $\mathrm{R}_{\mathrm{t}}$ representing the Taylor expansion residuals and $\delta=\theta \gamma$. If residuals, $\eta_{t}$, are serially correlated, then Equation (21) can be augmented to correct the serial correlation as follows:

$$
\Delta \bar{\omega}_{t}=\delta \bar{\omega}_{t-1}^{3}+\sum_{j=1}^{p} \psi_{j} \Delta \bar{\omega}_{t-j}+\eta_{t}
$$

We can collect the $\mathrm{t}$-statistics for $\delta=0$ against $\delta<0$ as follows:

$$
t_{i}^{K S S F F}(k)=\frac{\hat{\delta}}{\text { S.e. }(\hat{\delta})}, i=\mu, \tau
$$

where $\hat{\delta}$ is the OLS estimator of $\delta$ and s.e. $(\hat{\delta})$ is the standard error of $\hat{\delta}$.

To obtain the asymptotic distribution of the $t_{i}^{F K S S}(k)(i=\mu, \tau)$ test, we need the subsequent outcomes, where we let $[r T], r \in[0,1]$, be an integer close to $r T$. During the derivation $\rightarrow$ implies weak convergence as $T$ approaches $\infty$.

\section{Proposition 1.}

(i) $\quad T^{-3 / 2} \sum_{t=1}^{T} \bar{\omega}_{t} \rightarrow \sigma \int_{0}^{1} W(r) d r=\sigma f_{1}$

(ii) $\quad T^{-5 / 2} \sum_{t=1}^{T} t \bar{\omega}_{t} \rightarrow \sigma \int_{0}^{1} r W(r) d r=\sigma f_{2}$

(iii) $\quad T^{-3 / 2} \sum_{t=1}^{T} \sin \left(\frac{2 \pi k^{f r} t}{T}\right) \bar{\omega}_{t} \rightarrow \sigma \int_{0}^{1} \sin \left(2 \pi k^{f r} r\right) W(r) d r=\sigma f_{3}$

(iv) $\quad T^{-3 / 2} \sum_{t=1}^{T} \cos \left(\frac{2 \pi k k^{f r} t}{T}\right) \bar{\omega}_{t} \rightarrow \sigma \int_{0}^{1} \cos \left(2 \pi k^{f r} r\right) W(r) d r=\sigma f_{4}$

(v) $\quad T^{-1} \sum_{t=1}^{T} \sin \left(\frac{2 \pi k^{f r t}}{T}\right) \rightarrow \frac{1-\cos \left(2 \pi k^{f r}\right)}{2 \pi k^{f r}} \equiv s_{0}$

(vi) $\quad T^{-1} \sum_{t=1}^{T} \cos \left(\frac{2 \pi k^{f r} t}{T}\right) \rightarrow \frac{\sin \left(2 \pi k^{f r}\right)}{2 \pi k^{f r}} \equiv c_{0}$

(vii) $\quad T^{-2} \sum_{t=1}^{T} t \sin \left(\frac{2 \pi k^{f r} t}{T}\right) \rightarrow \frac{\sin \left(2 \pi k^{f r}\right)}{\left(2 \pi k^{f r}\right)^{2}}-\frac{\cos \left(2 \pi k^{f r}\right)}{2 \pi k^{f r}} \equiv s_{1}$

(viii) $\quad T^{-2} \sum_{t=1}^{T} t \cos \left(\frac{2 \pi k^{f r t}}{T}\right) \rightarrow \frac{\cos \left(2 \pi k^{f r}\right)-1}{\left(2 \pi k^{f r}\right)^{2}}-\frac{\sin \left(2 \pi k^{f r}\right)}{2 \pi k^{f r}} \equiv c_{1}$

(ix) $\quad T^{-1} \sum_{t=1}^{T} \sin ^{2}\left(\frac{2 \pi k^{f r_{t}}}{T}\right) \rightarrow \frac{1}{2}\left[1-\frac{\sin \left(4 \pi k^{f r}\right)}{4 \pi k^{f r}}\right] \equiv s_{2}$

(x) $\quad T^{-1} \sum_{t=1}^{T} \cos ^{2}\left(\frac{2 \pi k^{f r} t}{T}\right) \rightarrow \frac{1}{2}\left[1+\frac{\sin \left(4 \pi k^{f r}\right)}{4 \pi k^{f r}}\right] \equiv c_{2}$

(xi) $\quad T^{-1} \sum_{t=1}^{T} \sin \left(\frac{2 \pi k^{f r} t}{T}\right) \cos \left(\frac{2 \pi k^{f r} t}{T}\right) \rightarrow \frac{1-\cos \left(4 \pi k^{f r}\right)}{8 \pi k^{f r}} \equiv m_{0}$

(xii) $\quad \frac{1}{T^{2}} \sum_{t=1}^{T} \bar{\omega}_{t-1}^{3} \varepsilon_{t} \rightarrow \sigma^{4} \int_{0}^{1} W_{i}\left(k^{f r}, r\right)^{3} d W(r)$

(xiii) $\frac{1}{T^{4}} \sum_{t=1}^{T} \bar{\omega}_{t-1}^{6} \rightarrow \sigma^{6} \int_{0}^{1} W_{i}\left(k^{f r}, r\right)^{6} d r$ 
Theorem 1. Under the null hypothesis of unit root, the asymptotic distribution of $t^{K S S F F}$ is as follows:

$$
t_{i}^{K S S F F}\left(k^{f r}\right) \stackrel{d}{\rightarrow} \frac{\int_{0}^{1} W_{i}\left(k^{f r}, r\right)^{3} d W(r)}{\left(\int_{0}^{1} W_{i}\left(k^{f r}, r\right)^{6} d r\right)^{1 / 2}}, i=\mu, \tau
$$

where $W(r)$ is the Wiener process as defined over the interval $r \in[0,1] . W_{i}\left(k^{f r}, r\right)$ for $i=\mu, \tau$ is a demeaned and de-trended Brownian motion, respectively.

Proof of Theorem 1. See Appendix A.

Apparently, the asymptotic distribution of the subsequent test statistics under the null depends on the fractional Fourier frequency $k^{f r}$, however invariant to other parameters in the model. Therefore, the importance of frequency estimation in ESTAR fractional frequency (1) test is shown explicitly by deriving the asymptotic distribution.

Asymptotic critical values of the KSS-FF statistics for the intercept only and the intercept and trend cases have been arranged via simulations with $\mathrm{T}=2000$ and for 100000 replications. Critical values of the mentioned cases are displayed in Tables 2 and 3 (For critical values for the one step intercept only and the one step intercept and trend cases, see Appendix B).

Table 2. Critical values for the two step intercept only case.

\begin{tabular}{cccccccc}
\hline $\mathbf{k}=\mathbf{1}$ & $\mathbf{\% 1}$ & $\mathbf{9 5}$ & $\mathbf{\% 1 0}$ & $\mathbf{k}=\mathbf{2}$ & $\mathbf{\% 1}$ & $\mathbf{\% 5}$ & $\mathbf{\% 1 0}$ \\
\hline 1.1 & -4.103 & -3.522 & -3.212 & 2.1 & -3.853 & -3.272 & -2.989 \\
1.2 & -4.055 & -3.461 & -3.142 & 2.2 & -3.825 & -3.253 & -2.954 \\
1.3 & -4.017 & -3.403 & -3.077 & 2.3 & -3.818 & -3.230 & -2.931 \\
1.4 & -3.965 & -3.353 & -3.036 & 2.4 & -3.795 & -3.201 & -2.904 \\
1.5 & -3.940 & -3.341 & -3.019 & 2.5 & -3.756 & -3.172 & -2.876 \\
1.6 & -3.910 & -3.328 & -3.016 & 2.6 & -3.730 & -3.159 & -2.861 \\
1.7 & -3.901 & -3.323 & -3.015 & 2.7 & -3.722 & -3.151 & -2.848 \\
1.8 & -3.894 & -3.320 & -3.014 & 2.8 & -3.722 & -3.141 & -2.845 \\
1.9 & -3.868 & -3.302 & -3.011 & 2.9 & -3.721 & -3.140 & -2.845 \\
\hline $\mathrm{k}=3$ & & & & $\mathrm{k}=4$ & & & \\
\hline 3.1 & -3.707 & -3.139 & -2.839 & 4.1 & -3.632 & -3.067 & -2.777 \\
3.2 & -3.683 & -3.117 & -2.825 & 4.2 & -3.630 & -3.065 & -2.776 \\
3.3 & -3.677 & -3.102 & -2.807 & 4.3 & -3.630 & -3.066 & -2.772 \\
3.4 & -3.667 & -3.096 & -2.804 & 4.4 & -3.619 & -3.057 & -2.768 \\
3.5 & -3.665 & -3.089 & -2.795 & 4.5 & -3.617 & -3.044 & -2.760 \\
3.6 & -3.658 & -3.079 & -2.789 & 4.6 & -3.616 & -3.042 & -2.753 \\
3.7 & -3.652 & -3.078 & -2.786 & 4.7 & -3.615 & -3.041 & -2.752 \\
3.8 & -3.642 & -3.072 & -2.781 & 4.8 & -3.609 & -3.041 & -2.750 \\
3.9 & -3.640 & -3.067 & -2.778 & 4.9 & -3.607 & -3.040 & -2.750 \\
\hline $\mathrm{k}=5$ & & & & $\mathrm{k}=6$ & & & \\
\hline 5.1 & -3.605 & -3.040 & -2.749 & 6.1 & -3.572 & -3.020 & -2.721 \\
5.2 & -3.597 & -3.039 & -2.749 & 6.2 & -3.571 & -3.019 & -2.720 \\
5.3 & -3.595 & -3.035 & -2.741 & 6.3 & -3.570 & -3.018 & -2.719 \\
5.4 & -3.592 & -3.030 & -2.738 & 6.4 & -3.570 & -3.017 & -2.719 \\
5.5 & -3.591 & -3.027 & -2.734 & 6.5 & -3.568 & -3.016 & -2.718 \\
5.6 & -3.577 & -3.021 & -2.732 & 6.6 & -3.564 & -3.012 & -2.715 \\
5.7 & -3.573 & -3.018 & -2.722 & 6.7 & -3.563 & -3.010 & -2.715 \\
5.8 & -3.572 & -3.017 & -2.721 & 6.8 & -3.560 & -3.005 & -2.711 \\
5.9 & -3.572 & -3.017 & -2.721 & 6.9 & -3.544 & -3.000 & -2.708 \\
\hline & & & & & & & \\
\hline
\end{tabular}

Note: the asymptotic critical values are obtained by using 100,000 Monte Carlo trials and $\mathrm{T}=2000$. 
Table 3. Critical values for the two step intercept and trend case.

\begin{tabular}{cccccccc}
\hline $\mathbf{k}=\mathbf{1}$ & $\mathbf{\% 1}$ & $\mathbf{\% 5}$ & $\mathbf{\% 1 0}$ & $\mathbf{k}=\mathbf{2}$ & $\mathbf{\% 1}$ & $\mathbf{\% 5}$ & $\mathbf{\% 1 0}$ \\
\hline 1.1 & -4.639 & -4.092 & -3.815 & 2.1 & -4.363 & -3.795 & -3.502 \\
1.2 & -4.636 & -4.092 & -3.815 & 2.2 & -4.357 & -3.778 & -3.481 \\
1.3 & -4.628 & -4.084 & -3.799 & 2.3 & -4.322 & -3.757 & -3.464 \\
1.4 & -4.577 & -4.034 & -3.748 & 2.4 & -4.307 & -3.737 & -3.447 \\
1.5 & -4.556 & -3.986 & -3.700 & 2.5 & -4.294 & -3.723 & -3.417 \\
1.6 & -4.511 & -3.952 & -3.658 & 2.6 & -4.287 & -3.715 & -3.405 \\
1.7 & -4.486 & -3.916 & -3.617 & 2.7 & -4.250 & -3.676 & -3.375 \\
1.8 & -4.449 & -3.881 & -3.585 & 2.8 & -4.246 & -3.666 & -3.366 \\
1.9 & -4.422 & -3.854 & -3.558 & 2.9 & -4.241 & -3.659 & -3.354 \\
\hline $\mathrm{k}=3$ & & & & $\mathrm{k}=4$ & & & \\
\hline 3.1 & -4.204 & -3.632 & -3.336 & 4.1 & -4.112 & -3.571 & -3.274 \\
3.2 & -4.211 & -3.629 & -3.333 & 4.2 & -4.114 & -3.557 & -3.269 \\
3.3 & -4.169 & -3.611 & -3.323 & 4.3 & -4.106 & -3.556 & -3.268 \\
3.4 & -4.192 & -3.621 & -3.323 & 4.4 & -4.106 & -3.556 & -3.267 \\
3.5 & -4.176 & -3.612 & -3.321 & 4.5 & -4.125 & -3.561 & -3.267 \\
3.6 & -4.177 & -3.606 & -3.311 & 4.6 & -4.126 & -3.559 & -3.266 \\
3.7 & -4.159 & -3.589 & -3.295 & 4.7 & -4.127 & -3.558 & -3.263 \\
3.8 & -4.143 & -3.564 & -3.276 & 4.8 & -4.105 & -3.536 & -3.250 \\
3.9 & -4.141 & -3.571 & -3.275 & 4.9 & -4.100 & -3.538 & -3.250 \\
\hline $\mathrm{k}=5$ & & & & $\mathrm{k}=6$ & & & \\
\hline 5.1 & -4.076 & -3.519 & -3.233 & 6.1 & -4.062 & -3.500 & -3.214 \\
5.2 & -4.083 & -3.515 & -3.232 & 6.2 & -4.063 & -3.505 & -3.212 \\
5.3 & -4.104 & -3.528 & -3.231 & 6.3 & -4.053 & -3.494 & -3.211 \\
5.4 & -4.071 & -3.512 & -3.230 & 6.4 & -4.033 & -3.495 & -3.207 \\
5.5 & -4.079 & -3.515 & -3.230 & 6.5 & -4.045 & -3.489 & -3.206 \\
5.6 & -4.075 & -3.515 & -3.229 & 6.6 & -4.046 & -3.487 & -3.206 \\
5.7 & -4.075 & -3.519 & -3.228 & 6.7 & -4.062 & -3.487 & -3.204 \\
5.8 & -4.070 & -3.515 & -3.223 & 6.8 & -4.032 & -3.487 & -3.204 \\
5.9 & -4.074 & -3.496 & -3.218 & 6.9 & -4.053 & -3.484 & -3.197 \\
\hline
\end{tabular}

Note: The asymptotic critical values are obtained by using 100,000 Monte Carlo trials and $\mathrm{T}=2000$.

\subsection{Small Sample Properties of the Proposed Tests}

We use the following data-generating process for the size analysis:

$$
y_{t}=y_{t-1}+v_{t}
$$

As it is displayed in Table 4, no size problem is found in the critical values.

Table 4. Critical values for the two step intercept and trend case.

\begin{tabular}{ccccc}
\hline & $\mathbf{k}=\mathbf{1 . 1}$ & $\mathbf{k}=\mathbf{1 . 5}$ & $\mathbf{k}=\mathbf{2 . 1}$ & $\mathbf{k}=\mathbf{6 . 9}$ \\
\hline 100 & 0.051 & 0.052 & 0.053 & 0.050 \\
200 & 0.049 & 0.050 & 0.049 & 0.048 \\
500 & 0.050 & 0.051 & 0.048 & 0.047 \\
\hline
\end{tabular}

Note: The size analysis is conducted using 10,000 Monte Carlo trials.

For the power analysis, we have used the following DGP for the two-step representation:

$$
\begin{gathered}
\bar{\omega}_{t}=y_{t}-\hat{\alpha}_{0}-\hat{\alpha}_{1} \sin \left(\frac{2 \pi \hat{k}^{f r} t}{T}\right)-\hat{\alpha}_{2} \cos \left(\frac{2 \pi \hat{k}^{f r} t}{T}\right) \\
\Delta \bar{\omega}_{t}=\theta \bar{\omega}_{t-1}\left(1-\exp \left(-\gamma \bar{\omega}_{t-1}^{2}\right)\right)+\varepsilon_{t}
\end{gathered}
$$


and

$$
y_{t}=\hat{\alpha}_{0}+\hat{\alpha}_{1} \sin \left(\frac{2 \pi \hat{k}^{f r} t}{T}\right)+\hat{\alpha}_{2} \cos \left(\frac{2 \pi \hat{k}^{f r} t}{T}\right)+\theta \bar{\omega}_{t-1}\left(1-\exp \left(-\gamma \bar{\omega}_{t-1}^{2}\right)\right)+u_{t}
$$

for the one step approach.

The parameter regions are given in Table 5 as follows:

Table 5. Power analysis for the ESTAR (1) DGP.

\begin{tabular}{|c|c|c|c|c|c|}
\hline$\gamma$ & $\phi$ & $t_{N L}$ & $t^{F K S S}$ & $\begin{array}{c}t^{K S S F F} \\
\max k^{f}=2.0\end{array}$ & $\begin{array}{c}t^{K S S F F} \\
\max k^{f=5.0}\end{array}$ \\
\hline 0.01 & -0.1 & 0.082 & 0.090 & 0.223 & 0.240 \\
\hline 0.01 & -0.15 & 0.097 & 0.099 & 0.246 & 0.251 \\
\hline 0.01 & -0.25 & 0.128 & 0.129 & 0.261 & 0.302 \\
\hline 0.01 & -0.5 & 0.219 & 0.169 & 0.321 & 0.395 \\
\hline 0.01 & -0.75 & 0.333 & 0.219 & 0.392 & 0.438 \\
\hline 0.01 & -1.0 & 0.455 & 0.273 & 0.464 & 0.512 \\
\hline 0.05 & -0.1 & 0.135 & 0.122 & 0.263 & 0.291 \\
\hline 0.05 & -0.15 & 0.201 & 0.155 & 0.302 & 0.370 \\
\hline 0.05 & -0.25 & 0.371 & 0.226 & 0.410 & 0.501 \\
\hline 0.05 & -0.5 & 0.760 & 0.468 & 0.608 & 0.726 \\
\hline 0.05 & -0.75 & 0.923 & 0.694 & 0.789 & 0.859 \\
\hline 0.05 & -1.0 & 0.976 & 0.846 & 0.907 & 0.939 \\
\hline 0.1 & -0.1 & 0.175 & 0.138 & 0.282 & 0.337 \\
\hline 0.1 & -0.15 & 0.289 & 0.188 & 0.352 & 0.426 \\
\hline 0.1 & -0.25 & 0.554 & 0.319 & 0.493 & 0.628 \\
\hline 0.1 & -0.5 & 0.925 & 0.699 & 0.800 & 0.867 \\
\hline 0.1 & -0.75 & 0.990 & 0.912 & 0.942 & 0.960 \\
\hline 0.1 & -1.0 & 0.998 & 0.978 & 0.985 & 0.993 \\
\hline 0.5 & -0.1 & 0.234 & 0.165 & 0.340 & 0.413 \\
\hline 0.5 & -0.15 & 0.415 & 0.253 & 0.426 & 0.566 \\
\hline 0.5 & -0.25 & 0.752 & 0.507 & 0.670 & 0.776 \\
\hline 0.5 & -0.5 & 0.991 & 0.943 & 0.960 & 0.992 \\
\hline 0.5 & -0.75 & 0.999 & 0.997 & 1.000 & 1.000 \\
\hline 0.5 & -1.0 & 1.000 & 0.999 & 1.000 & 1.000 \\
\hline 1.0 & -0.1 & 0.239 & 0.168 & 0.330 & 0.382 \\
\hline 1.0 & -0.15 & 0.418 & 0.263 & 0.467 & 0.542 \\
\hline 1.0 & -0.25 & 0.750 & 0.528 & 0.670 & 0.784 \\
\hline 1.0 & -0.5 & 0.991 & 0.951 & 0.972 & 0.992 \\
\hline 1.0 & -0.75 & 0.999 & 0.997 & 1.000 & 1.000 \\
\hline 1.0 & -1.0 & 1.000 & 1.000 & 1.000 & 1.000 \\
\hline
\end{tabular}

Note: The power analysis is obtained by using 100,000 Monte Carlo trials with $\mathrm{T}=100$.

As it is demonstrated in Table 5, the KSS-FF test is superior to the KSS test in almost all parameter regions. This power analysis is consistent with the simulation results given in Table 1. If we can capture the residuals of Taylor expansion with the Fourier function, then it becomes evident that the power of the KSS-FF test will be high. We have proved here that the KSS-FF test increases the power of ESTAR (1) type tests, which was aforementioned in Section 2.1. More importantly, the highest power gain of the KSS-FF test is in the region that represents the nature of the financial data. As we mentioned in the introduction part, many financial data have a nonlinear structure with high persistence. This parameter range manifests itself in Table 5 with the 0.01 and 0.05 gamma parameter range and -0.1 and -0.5 phi parameter range. The drawings of these ranges (in 3D) and their projections (in 2D) are given in Figure 4. Accordingly, we see that the power gain of the KSS-FF test increases in the regions that display high persistence and nonlinearity. This result is a significant contribution to the literature. It prevents the researchers from misleadingly concluding that financial data contain unit roots. In fact, by decomposing the stochastic properties of financial variables correctly, the near unit root problem is prevented. 

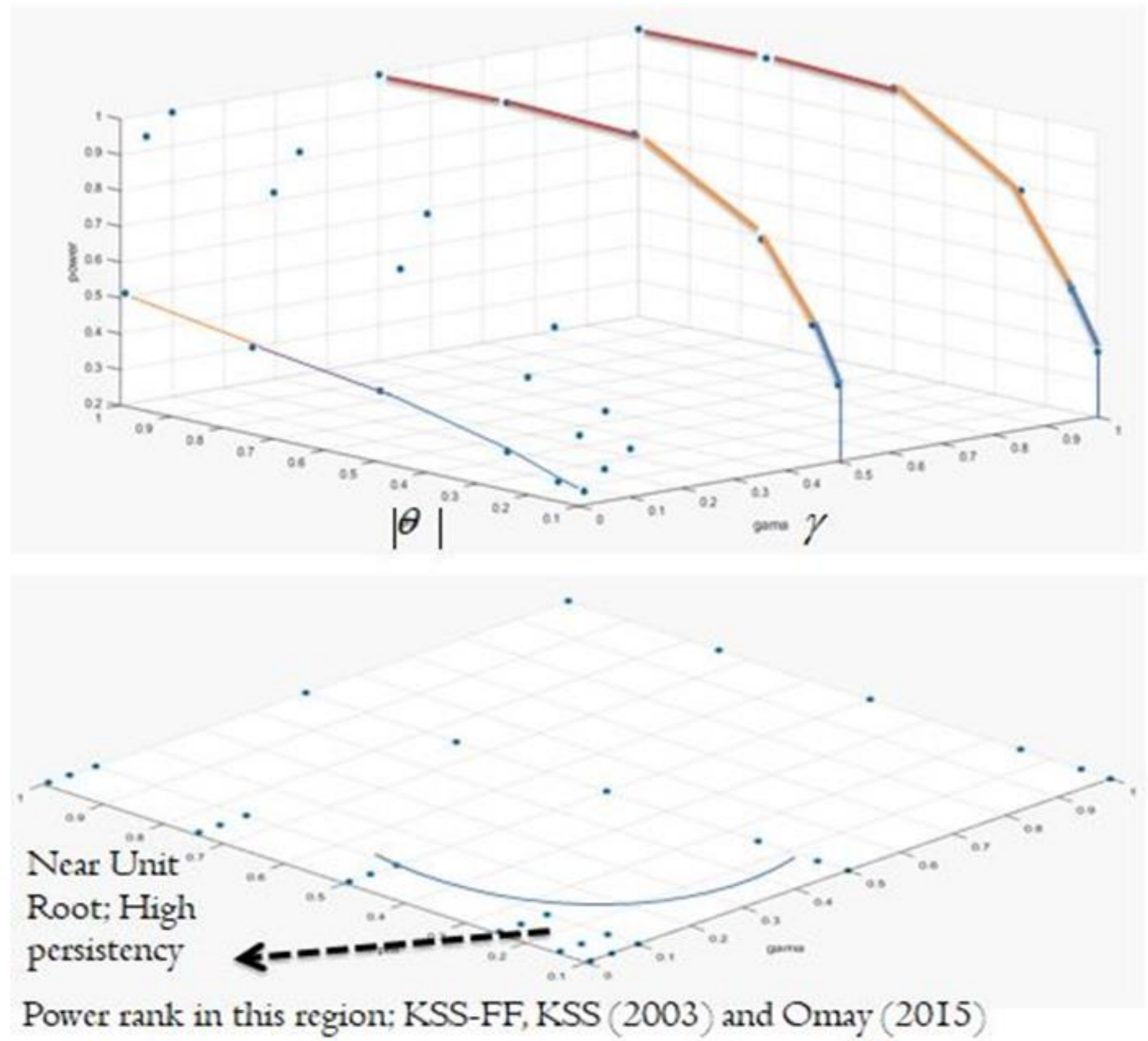

Figure 4. Power of the test and the region where the financial data are located: the panel on the top shows the power analysis in the 3D diagram, where the results are obtained by using alternative values of $\gamma$ and $\theta$. The power results located on the vertical axis change from high power colored by red to low power colored by blue. As the values of $\gamma$ and $\theta$ increase, the power of the test increases as well. In the near unit root high-persistence area, the KSS-FF test proposed in our study becomes the most powerful test. The power of the KSS-FF test is already much higher than the other tests in other areas. That is, because financial variables exhibit the structure in the near unit root persistence area, one can say that the proposed KSS-FF statistic is more suitable to test for the stochastic properties of financial variables. This can be seen in the blue hyperbolic area of the projective graph at the bottom.

\section{Limits of the Fourier Function in the Framework of the ESTAR Unit Root Test}

In which case can the Fourier function be taken to represent multiple smooth breaks? To answer this question, we look at the power analysis results of the KSS-FF DGP. If the power of Fourier unit root test becomes worse than that of the KSS-FF test, it can be said that it has captured the break in that parameter regions. We can decide this from the power analysis given in Table 6.

When KSS-FF is greater than FFFFF, parameter values of that region can be defined as smooth transitional structural break, apart from ESTAR type unit root test. As can be seen from Table 6, the power of the FFFFF test is higher than the KSS-FF test in almost all parameter fields. These findings displayed in Table 6 are similar to the results of [16] for the integer form frequency Fourier KSS test. When we take the amplitude parameters of sine and cosine as equal, the power of the KSS-FF test increases which is consistent with the results of [15], and it passes the power of the FFFFF test at $\gamma=0.01$ and $\phi=0.75$ in only one parameter region. Integer form FKSS test was superior to FADF test in this field. However, it is understood that this situation disappears when the frequency becomes fractional. 
These results prove the accuracy of the simulation study results given in Section 2. The use of the variable $y_{t-1}^{3}$ obtained from the expansion of the ESTAR function with the Taylor methodology captures the Taylor expansion residuals only. In this sense, the KSS-FF test is only a state-dependent test and does not provide any superiority over the FFFFF test against any structural break.

Table 6. Power analysis with KSS-FF DGP.

\begin{tabular}{|c|c|c|c|c|c|c|c|}
\hline$\alpha_{1}$ & $\alpha_{2}$ & $\gamma$ & $\phi$ & $\begin{array}{c}\text { Omay (2015) } \\
t^{F F F F F} \\
k^{f}=1.5\end{array}$ & $t_{N L} \mathrm{KSS}$ & $t^{F K S S} \mathrm{CL}$ & $\begin{array}{l}t^{K S S F F} \\
k^{f}=1.5\end{array}$ \\
\hline 0 & 5 & 0.01 & -0.1 & 0.330 & 0.001 & 0.002 & 0.126 \\
\hline 3 & 0 & 0.01 & -0.1 & 0.089 & 0.021 & 0.011 & 0.071 \\
\hline 3 & 5 & 0.01 & -0.1 & 0.356 & 0.000 & 0.002 & 0.124 \\
\hline 0 & 5 & 0.01 & -0.75 & 0.638 & 0.001 & 0.002 & 0.201 \\
\hline 3 & 0 & 0.01 & -0.75 & 0.222 & 0.046 & 0.015 & 0.161 \\
\hline 3 & 5 & 0.01 & -0.75 & 0.675 & 0.000 & 0.002 & 0.203 \\
\hline 0 & 5 & 0.5 & -0.1 & 0.678 & 0.001 & 0.002 & 0.228 \\
\hline 3 & 0 & 0.5 & -0.1 & 0.237 & 0.046 & 0.021 & 0.108 \\
\hline 3 & 5 & 0.5 & -0.1 & 0.701 & 0.000 & 0.002 & 0.247 \\
\hline 0 & 5 & 0.5 & -0.75 & 1.000 & 0.077 & 0.003 & 0.992 \\
\hline 3 & 0 & 0.5 & -0.75 & 1.000 & 0.802 & 0.449 & 0.964 \\
\hline 3 & 5 & 0.5 & -0.75 & 1.000 & 0.011 & 0.002 & 0.982 \\
\hline 0 & 5 & 1.0 & -0.1 & 0.695 & 0.002 & 0.002 & 0.233 \\
\hline 3 & 0 & 1.0 & -0.1 & 0.257 & 0.052 & 0.019 & 0.151 \\
\hline 3 & 5 & 1.0 & -0.1 & 0.723 & 0.000 & 0.002 & 0.247 \\
\hline 0 & 5 & 1.0 & -0.75 & 1.000 & 0.115 & 0.002 & 1.000 \\
\hline 3 & 0 & 1.0 & -0.75 & 1.000 & 0.887 & 0.593 & 0.990 \\
\hline 3 & 5 & 1.0 & -0.75 & 1.000 & 0.021 & 0.003 & 0.993 \\
\hline \multicolumn{8}{|c|}{ Equal amplitude parameter. } \\
\hline 1 & 1 & 0.01 & -0.1 & 0.093 & 0.058 & 0.021 & 0.085 \\
\hline 1 & 1 & 0.01 & -0.5 & 0.193 & 0.159 & 0.045 & 0.187 \\
\hline 1 & 1 & 0.01 & -0.75 & 0.245 & 0.227 & 0.061 & 0.302 \\
\hline 1 & 1 & 0.5 & -0.1 & 0.262 & 0.146 & 0.029 & 0.177 \\
\hline 1 & 1 & 0.5 & -0.75 & 1.000 & 0.982 & 0.695 & 0.999 \\
\hline 1 & 1 & 1.0 & -0.1 & 0.268 & 0.138 & 0.024 & 0.186 \\
\hline 1 & 1 & 1.0 & -0.75 & 1.000 & 0.992 & 0.792 & 0.994 \\
\hline
\end{tabular}

\section{Empirical Analysis: Real Interest Rate Data of 11 OECD Countries}

In this section we will apply the newly developed KSS-FF test to the real interest rate series of the OECD countries as done in Kapetanios et al. (2003) [11] and show its advantage over the KSS test. Ref. [11] test for the presence of the unit roots in the nonlinear environment faced by financial markets using ex-post real interest rates and claim that the test that they propose will capture this nonlinear behavior in the case of a near unit root. The authors emphasize that the apparent unit root behavior in ex-post interest rates has become a strange puzzle for economists. They argue that transaction costs in financial asset markets are likely to lead to nonlinear rates of convergence to the equilibrium rates of return. The Fisher hypothesis predicts that the long-run equilibrium value of the real interest rates remains around a common constant value. The apparent stationarity of real interest rates clearly violates this hypothesis, which creates the interest rate paradox mentioned above.

Due to the presence of transaction costs and other market frictions, it is entirely plausible that the financial variables deviate from their equilibrium values the greater the investment/arbitrage adjustment flows that pull them back. If so, the results in this article show that the failure to reject the unit root null may be due to a lack of power of the tests used. Ref. [11] also claim that the conventionally used ADF test has a lack of power in testing unit root in nonlinear structures, while we show that the use of the linearized 
auxiliary regression equation of the KSS test would produce a similar result and its power will be low. In this study, we recompile the data set that [11] have used for eleven major OECD economies. Then, we apply the KSS test and our newly proposed KSS-FF test to the ex-post real interest rates of these eleven major OECD economies. In this way, our aim is to show that our newly proposed method solves the low power problem by capturing the nonlinear behavior in the near unit root region better.

For the empirical application, the quarterly real interest rate data of Australia (AU), Canada (CA), France (FR), Germany (GE), Italy (IT), Japan (JA), Netherlands (NE), New Zealand (NZ), Spain (SP), United Kingdom (UK) and United States (US) were calculated by taking the difference between the respective nominal interest rates and inflation rates Nominal interest rates and Consumer Price Index (CPI) data were retrieved from the International Financial Statistics (IFS) database (https:/ / data.imf.org/?sk=4C514D48-B6 BA-49ED-8AB9-52B0C1A0179B\&sId=1409151240976, accessed on 19 July 2021).The real interest rate data are seasonally adjusted.

Nominal interest rate series used are the treasury bill rates for CA, JA, UK, US and NZ, money market rate for GE, SP, NE and IT, central bank policy rate for AU and deposit rate for FR. The sample period has been determined according to the availability of data. The sample period is 1957: Q1-2000: Q4 for CA, JA, UK and US; 1960: Q1-2000: Q4 for GE, NE; 1966: Q1-2000: Q4 for FR, 1960: Q3-2000: Q4 for AU; 1971: Q1-2000: Q4 for IT; 1974: Q1-2000: Q4 for SP and 1978: Q1-2000: Q4 for NZ (Nominal interest rates of NZ between 1985: Q3 and 1986: Q1 were missing in the IFS; therefore, these three data points were obtained from OECD website:OECD (2021), short-term interest rates (indicator). doi:10.1787/2cc37d77-en, accessed on 19 July 2021.

The results of the empirical study are displayed in Table 7.

Table 7. Eleven OECD real interest rates.

\begin{tabular}{cccc}
\hline Country & KSS & KSS-FF & Selected Frequency \\
\hline AU & $-1.576^{* *}$ & $-3.044^{*}$ & 1.0 \\
CA & $-2.724^{* *}$ & $-4.369^{*}$ & 1.1 \\
FR & $-6.708^{*}$ & $-7.270^{*}$ & 1.4 \\
GE & $-3.478^{* *}$ & $-3.685^{* *}$ & 1.0 \\
IT & $-3.437^{* *}$ & $-4.648^{*}$ & 1.0 \\
JA & $-2.392^{* *}$ & $-3.919^{* *}$ & 1.1 \\
NE & $-3.088^{* *}$ & $-4.454^{*}$ & 0.1 \\
NZ & $-4.043^{*}$ & $-5.176^{*}$ & 0.5 \\
SP & $-3.474^{* *}$ & $-4.937^{*}$ & 1.2 \\
UK & $-2.113^{*}$ & $-3.522^{*}$ & 2.4 \\
US & $-1.988^{*}$ & $-4.355^{*}$ & \\
\hline
\end{tabular}

Note: The $t_{N L}$ and $t^{K S S F F}$ statistics are computed using the de-meaned data in a regression model with a maximum of 8 augmentations, where the insignificant augmentation terms in a companion AR (8) model for $\Delta y_{t}$ were excluded. In all cases ${ }^{*}{ }^{* *}$ and ${ }^{* * *}$ denote significance level at $1 \%, 5 \%$ and $10 \%$.

As it can be seen from Table 7, both tests fail to reject the unit root null for AU, which means that the real interest rate data of AU does not exhibit ESTAR type of nonlinear stationarity between 1960: Q3 and 2000: Q4. The null hypothesis of unit root can be rejected for GE between 1960: Q1 and 2000: Q4, for FR between 1966: Q1 and 2000: Q4 and for NZ between 1978: Q1 and 2000: Q4 by both tests at the same significance level. For CA between 1957: Q1 and 2000: Q4, for IT between 1971: Q1 and 2000: Q4, for NE between 1960: Q1 and 2000: Q4 and for SP between 1974: Q1 and 2000: Q4, the null of unit root is rejected but the KSS-FF test is more significant than the KSS test. According to the results given in Table 7, we cannot detect ESTAR type of nonlinearity for JA and US between 1957: Q1 and 2000: Q4 and for SP between 1974: Q1 and 2000: Q4 by using the KSS-FF test, since the KSS test fails to reject the unit root null. These results are consistent with the simulation studies we have shown since the beginning of the study. The KSS-FF test takes the Taylor expansion, which is also considered in the KSS test, one step further and 
increases the power of the test significantly, as it predicts the original function in the case of a near unit root. The test statistics were found more significant for all countries in the case of the KSS-FF test than the KSS test. Generally, these outcomes promote the KSS-FF test as a suitable complementary statistic to employ in standard ESTAR unit root testing, notably when the series is recognized to be highly persistent but is anticipated a priori to be stationary. The estimates also suggest that the KSS-FF model itself may provide a better alternative to a KSS model in such cases.

\section{Conclusions}

Stationarity has been an integral part of time series econometrics. If the series is not stationary, the obtained parameter results pose a spurious regression problem. However, the literature on testing the stationarity of the economic time series had focused intensively on the linear unit root testing procedures until the study of [11], and possible nonlinearities in the time series were ignored. The main contribution of [11] to the literature is developing a new unit root test statistic designed to be more robust against stationary ESTAR processes. In this sense, KSS test was one of the first studies to detect stationarity not only in linear but also in nonlinear time series. However, as we stated throughout this study, the weakness of the KSS test is that the ESTAR process is approximated with a Taylor expansion. The approximation of the functional structure of the KSS test leads to a decrease in the power of the test. Thus, it leads to misleading conclusions about the stationarity of especially financial data exhibiting nonlinearities and having near unit root problems. To overcome this, we propose the use of the KSS-FF test, which allows for state-dependent nonlinear structures with near unit roots to be tested more precisely. The empirical study on ex-post real interest rates of 11 OECD countries reveals the importance of recovering the information loss, which is achieved by adding the fractional Fourier function to the deterministic component of the ESTAR (1) test. In this regard, we have contributed to the literature by producing the most accurate ESTAR (1) test. For further research, other financial variables can also be analyzed using the KSS-FF test and the economic data that exhibit these hybrid nonlinear structures can be determined.

Author Contributions: Conceptualization, T.O. and A.C.; methodology, T.O.; software, T.O.; validation, A.C.; formal analysis, T.O.; investigation, T.O. and A.C.; data curation, A.C. and E.H.; writing-original draft, A.C. and E.H.; writing—review and editing, A.C. and E.H.; visualization, T.O. and E.H.; supervision, T.O. and A.C. All authors have read and agreed to the published version of the manuscript.

Funding: This research received no external funding.

Institutional Review Board Statement: Not applicable.

Informed Consent Statement: Not applicable.

Data Availability Statement: Publicly available data were obtained for this study. Nominal interest rates and CPI data can be found here: https: / / data.imf.org / ?sk=4C514D48-B6BA-49ED-8AB9-5 2B0C1A0179B\&sId=1409151240976, accessed on 19 July 2021. Nominal interest rates of NZ data are available in a publicly accessible repository: OECD (2021), short-term interest rates (indicator). doi:10.1787/2cc37d77-en, accessed on 19 July 2021.

Conflicts of Interest: The authors declare no conflict of interest.

\section{Appendix A}

\section{Proof of Proposition 1.}

The proofs of (i) and (ii) are known (see Hamilton, 1994 [24]) (p. 486). By using the continuous mapping theorem, we can attain the proofs of (iii) and (iv) as follows: 


$$
\begin{aligned}
& T^{-3 / 2} \sum_{t=1}^{T} \sin \left(\frac{2 \pi k t}{T}\right) \bar{\omega}_{t} \rightarrow \sigma \int_{0}^{1} \sin (2 \pi k r) W(r) d r \equiv \sigma f_{3} \\
& T^{-3 / 2} \sum_{t=1}^{T} \cos \left(\frac{2 \pi k t}{T}\right) \bar{\omega}_{t} \rightarrow \sigma \int_{0}^{1} \cos (2 \pi k r) W(r) d r=\sigma f_{4} \\
& T^{-1} \sum_{t=1}^{T} \sin \left(\frac{2 \pi k^{f r} t}{T}\right) \rightarrow \int_{0}^{1} \sin \left(2 \pi k^{f r} r\right) d r=\frac{1-\cos \left(2 \pi k^{f r}\right)}{2 \pi k^{f r}} \equiv s_{0} \\
& T^{-1} \sum_{t=1}^{T} \cos \left(\frac{2 \pi k^{f r} t}{T}\right) \rightarrow \int_{0}^{1} \cos \left(2 \pi k^{f r} r\right) d r=\frac{\sin \left(2 \pi k^{f r}\right)}{2 \pi k^{f r}} \equiv c_{0} \\
& T^{-2} \sum_{t=1}^{T} t \sin \left(\frac{2 \pi k^{f r} t}{T}\right) \rightarrow \int_{0}^{1} r \sin \left(2 \pi k^{f r} r\right) d r=\frac{\sin \left(2 \pi k^{f r}\right)}{\left(2 \pi k^{f r}\right)^{2}}-\frac{\cos \left(2 \pi k^{f r}\right)}{2 \pi k^{f r}} \equiv s_{1} \\
& T^{-2} \sum_{t=1}^{T} t \cos \left(\frac{2 \pi k^{f r} t}{T}\right) \rightarrow \int_{0}^{1} r \cos \left(2 \pi k^{f r} r\right) d r=\frac{\cos \left(2 \pi k^{f r}\right)-1}{\left(2 \pi k^{f r}\right)^{2}}+\frac{\sin \left(2 \pi k^{f r}\right)}{2 \pi k^{f r}} \equiv c_{1} \\
& T^{-1} \sum_{t=1}^{T} \sin ^{2}\left(\frac{2 \pi k^{f r} t}{T}\right) \rightarrow \int_{0}^{1} \sin ^{2}\left(2 \pi k^{f r} r\right) d r=\frac{1}{2} \int_{0}^{1}\left[1-\cos \left(4 \pi k^{f r} r\right)\right] d r=\frac{1}{2}-\frac{\sin \left(4 \pi k^{f r}\right)}{8 \pi k^{f r}} \equiv s_{2} \\
& T^{-1} \sum_{t=1}^{T} \cos ^{2}\left(\frac{2 \pi k^{f r} t}{T}\right) \rightarrow \int_{0}^{1} \cos ^{2}\left(2 \pi k^{f r} r\right) d r=\frac{1}{2} \int_{0}^{1}\left[1+\cos \left(4 \pi k^{f r} r\right)\right] d r=\frac{1}{2}+\frac{\sin \left(4 \pi k^{f r}\right)}{8 \pi k^{f r}} \equiv c_{2} \\
& T^{-1} \sum_{t=1}^{T} \sin \left(\frac{2 \pi k^{f r} t}{T}\right) \cos \left(\frac{2 \pi k^{f r} t}{T}\right) \rightarrow \int_{0}^{1} \sin \left(2 \pi k^{f r} r\right) \cos \left(2 \pi k^{f r} r\right) d r=\frac{1}{2} \int_{0}^{1} \sin \left(4 \pi k^{f r} r\right) d r= \\
& \frac{\left[1-\cos \left(4 \pi k^{f r}\right)\right]}{8 \pi k^{f r}} \equiv m_{0}
\end{aligned}
$$

\section{Proof of Theorem 1}

Let $\sin (t)=\sin \left(2 \pi k^{f r} t / T\right)$ and $\cos (t)=\cos \left(2 \pi k^{f r} t / T\right)$. We first examine the de-meaned case with $\delta=0$ in Equation (21). Let $y_{t}^{\mu}$ denote the OLS residuals from the de-meaned case in the text with $w_{t}=(1, \sin (t), \cos (t))^{\prime}$

$$
y_{t}^{\mu}=\bar{\omega}_{t}-w_{t}^{\prime}(\hat{\theta}-\theta)
$$

where $\theta=\left(\alpha_{0}, \alpha_{1}, \alpha_{2}\right)^{\prime}, \hat{\theta}$ is the OLS estimator of $\theta$. We let $w=\left(w_{1}, \ldots, w_{T}\right)^{\prime}, \bar{\omega}=$ $\left(\bar{\omega}_{1}, \ldots, \bar{\omega}_{T}\right)^{\prime}$ and $\mathbf{M}_{T}=\operatorname{diag}(\sqrt{T}, \sqrt{T}, \sqrt{T})$ to have:

$$
\mathbf{M}_{T}(\hat{\theta}-\theta)=\left[\mathbf{M}_{T}^{-1} w^{\prime} w \mathbf{M}_{T}^{-1}\right]^{-1} \mathbf{M}_{T}^{-1} w^{\prime} \bar{\omega}
$$

Applying some algebra to (A1) and (A2),

$$
T^{-1 / 2} y_{[r T]}^{\mu}=T^{-1 / 2} \bar{\omega}_{[r T]}^{\mu}-T^{-1} w_{[r T]}^{\prime}\left[\mathbf{M}_{T}^{-1} w^{\prime} w \mathbf{M}_{T}^{-1}\right]^{-1} \mathbf{M}_{T}^{-1} w^{\prime} \bar{\omega}
$$

Depending on FCLT, the first term of (A3) becomes:

$$
T^{-1 / 2} \bar{\omega}_{[r T]}=T^{-1 / 2} \sum_{t=1}^{[r T]} \xi_{t} \rightarrow \sigma W(r)
$$

The second component in (A3) becomes: 


$$
\begin{aligned}
& {\left[\mathbf{M}_{T}^{-1} w^{\prime} w \mathbf{M}_{T}^{-1}\right]^{-1}=} \\
& {\left[\begin{array}{ccc}
1 & T^{-1} \sum_{t=1}^{T} \sin (t) & T^{-1} \sum_{t=1}^{T} \cos (t) \\
T^{-1} \sum_{t=1}^{T} \sin (t) & T^{-1} \sum_{t=1}^{T} \sin ^{2}(t) & T^{-1} \sum_{t=1}^{T} \sin (t) T^{-1} \sum_{t=1}^{T} \cos (t) \\
T^{-1} \sum_{t=1}^{T} \cos (t) & T^{-1} \sum_{t=1}^{T} \sin (t) T^{-1} \sum_{t=1}^{T} \cos (t) & T^{-1} \sum_{t=1}^{T} \cos ^{2}(t)
\end{array}\right]^{-1}} \\
& \rightarrow\left[\begin{array}{ccc}
1 & s_{0} & c_{0} \\
s_{0} & s_{2} & m_{0} \\
c_{0} & m_{0} & c_{2}
\end{array}\right]^{-1}=\frac{1}{\Delta_{1}}\left[\begin{array}{lll}
a_{11} & a_{12} & a_{13} \\
a_{12} & a_{22} & a_{23} \\
a_{13} & a_{23} & a_{33}
\end{array}\right]
\end{aligned}
$$

where $\Delta_{1}=s_{2} c_{2}-s_{2} c_{0}^{2}-s_{0}^{2} c_{2}-m_{0}^{2}, a_{11}=s_{2} c_{2}-m_{0}^{2}, a_{12}=c_{0} m_{0}-s_{0} c_{2}, a_{13}=s_{0} m_{0}-s_{2} c_{0}$, $a_{22}=c_{2}-c_{0}^{2}, a_{23}=s_{0} c_{0}-m_{0}$ and $a_{33}=s_{2}-s_{0}^{2}$.

$$
T^{-1} \mathbf{M}_{T}^{-1} w^{\prime} \bar{\omega}=\left[\begin{array}{c}
T^{-1 / 2} \sum_{t=1}^{T} \bar{\omega}_{t} \\
T^{-1 / 2} \sum_{t=1}^{T} \sin (t) \bar{\omega}_{t} \\
T^{-1 / 2} \sum_{t=1}^{T} \cos (t) \bar{\omega}_{t}
\end{array}\right] \rightarrow\left[\begin{array}{c}
\sigma f_{1} \\
\sigma f_{3} \\
\sigma f_{4}
\end{array}\right]
$$

Then,

$$
T^{-1} w_{[r T]}^{\prime}\left[\mathbf{M}_{T}^{-1} w^{\prime} w \mathbf{M}_{T}^{-1}\right]^{-1} \mathbf{M}_{T}^{-1} w^{\prime} \bar{\omega} \rightarrow \frac{1}{\Delta_{1}}\left[\begin{array}{ll}
1 & \sin \left(2 \pi k^{f r} r\right) \\
\cos \left(2 \pi k^{f r} r\right)
\end{array}\right]\left[\begin{array}{lll}
a_{11} & a_{12} & a_{13} \\
a_{21} & a_{22} & a_{23} \\
a_{31} & a_{32} & a_{33}
\end{array}\right]\left[\begin{array}{l}
\sigma f_{1} \\
\sigma f_{3} \\
\sigma f_{4}
\end{array}\right]
$$

Finally, merging the outcomes in Equations (A4) and (A7), we attain the de-meaned Brownian motion by

$$
\frac{1}{\sigma \sqrt{T}} y_{[r T]}^{u} \rightarrow W_{\mu}\left(k^{f r}, r\right)=W(r)-\frac{\sigma}{\Delta_{1}}\left[\begin{array}{c}
\left(a_{11} f_{1}+a_{12} f_{3}+a_{13} f_{4}\right) \\
+\left(a_{21} f_{1}+a_{22} f_{3}+a_{23} f_{4}\right) \sin \left(2 \pi k^{f r} r\right) \\
+\left(a_{31} f_{1}+a_{32} f_{3}+a_{33} f_{4}\right) \cos \left(2 \pi k^{f r} r\right)
\end{array}\right]
$$

For the de-trended case, similar arguments follow so we skip the same algebra. Using the above given results, under the null we can obtain the de-meaned Brownian's. Now we can proceed with the ESTAR (1) part in the second step. Under the null hypothesis, that is a random walk and applying Proposition 1.

Since we have arranged the de-meaned Brownian motions, we can combine them with the results of Kapetanios et al. (2003) [11]. For xii and xiii, see [11]. Following [11], it follows directly from the continuous mapping theorem, weak convergence of stochastic integrals and the semi-martingale property of $\varepsilon_{t}$ that

$$
\frac{1}{T^{2}} \sum_{t=1}^{T} \bar{\omega}_{t-1}^{3} \eta_{t} \rightarrow \sigma^{4} \int_{0}^{1} W_{i}\left(k^{f r}, r\right)^{3} d W(r)
$$

and

$$
\frac{1}{T^{4}} \sum_{t=1}^{T} \bar{\omega}_{t-1}^{6} \rightarrow \sigma^{6} \int_{0}^{1} W_{i}\left(k^{f r}, r\right)^{6} d r
$$


Therefore, we can conclude that the asymptotic distribution of KSS-FF is as follows:

$$
t_{i}^{K S S F F}\left(k^{f r}\right) \stackrel{d}{\rightarrow} \frac{\int_{0}^{1} W_{i}\left(k^{f r}, r\right)^{3} d W(r)}{\left(\int_{0}^{1} W_{i}\left(k^{f r}, r\right)^{6} d r\right)^{1 / 2}}, i=\mu, \tau
$$

Here, asymptotic distribution was obtained using the traditional de-meaned Brownian method. For critical values obtained in a single step, the asymptotic approach used in Cai and Omay (2021) [25] is more appropriate. Although there is a minimal difference between them, using demanded Brownian in terms of asymptotic distribution is more manageable in terms of derivation and more helpful as pedagogical means. See also Omay and Dumitru (2021) [26] for further derivations of the asymptotic distribution. Asymptotic distributions obtained in one step can also be provided for interested readers.

\section{Appendix B}

Table A1. Critical values for the one step intercept only case.

\begin{tabular}{cccccccc}
\hline $\mathbf{k}=\mathbf{1}$ & $\mathbf{0 1}$ & $\mathbf{9 5}$ & $\mathbf{9 1 0}$ & $\mathbf{k}=\mathbf{2}$ & $\mathbf{\% 1}$ & $\mathbf{\% 5}$ & $\mathbf{\% 1 0}$ \\
\hline 1.1 & -4.119 & -3.537 & -3.233 & 2.1 & -3.821 & -3.204 & -2.854 \\
1.2 & -4.105 & -3.512 & -3.204 & 2.2 & -3.819 & -3.189 & -2.877 \\
1.3 & -4.099 & -3.495 & -3.180 & 2.3 & -3.814 & -3.185 & -2.877 \\
1.4 & -4.029 & -3.439 & -3.122 & 2.4 & -3.798 & -3.159 & -2.835 \\
1.5 & -3.996 & -3.399 & -3.081 & 2.5 & -3.786 & -3.169 & -2.850 \\
1.6 & -3.963 & -3.358 & -3.043 & 2.6 & -3.749 & -3.156 & -2.828 \\
1.7 & -3.923 & -3.304 & -2.985 & 2.7 & -3.726 & -3.116 & -2.810 \\
1.8 & -3.865 & -3.261 & -2.948 & 2.8 & -3.722 & -3.123 & -2.797 \\
1.9 & -3.866 & -3.242 & -2.916 & 2.9 & -3.709 & -3.103 & -2.792 \\
\hline $\mathrm{k}=3$ & & & & $\mathrm{k}=4$ & & & \\
\hline 3.1 & -3.705 & -3.098 & -2.778 & 4.1 & -3.636 & -3.033 & -2.726 \\
3.2 & -3.689 & -3.070 & -2.772 & 4.2 & -3.625 & -3.028 & -2.724 \\
3.3 & -3.679 & -3.078 & -2.769 & 4.3 & -3.621 & -3.025 & -2.723 \\
3.4 & -3.677 & -3.077 & -2.765 & 4.4 & -3.619 & -3.023 & -2.719 \\
3.5 & -3.675 & -3.076 & -2.764 & 4.5 & -3.613 & -3.023 & -2.718 \\
3.6 & -3.675 & -3.068 & -2.751 & 4.6 & -3.611 & -3.020 & -2.718 \\
3.7 & -3.668 & -3.055 & -2.748 & 4.7 & -3.610 & -3.012 & -2.711 \\
3.8 & -3.640 & -3.043 & -2.741 & 4.8 & -3.595 & -3.012 & -2.707 \\
3.9 & -3.634 & -3.046 & -2.740 & 4.9 & -3.591 & -3.011 & -2.706 \\
\hline $\mathrm{k}=5$ & & & & $\mathrm{k}=6$ & & & \\
\hline 5.1 & -3.592 & -3.001 & -2.697 & 6.1 & -3.567 & -2.986 & -2.692 \\
5.2 & -3.587 & -2.999 & -2.696 & 6.2 & -3.567 & -2.985 & -2.684 \\
5.3 & -3.582 & -2.998 & -2.694 & 6.3 & -3.567 & -2.980 & -2.682 \\
5.4 & -3.578 & -2.996 & -2.692 & 6.4 & -3.563 & -2.976 & -2.680 \\
5.5 & -3.570 & -2.995 & -2.692 & 6.5 & -3.553 & -2.974 & -2.679 \\
5.6 & -3.567 & -2.993 & -2.690 & 6.6 & -3.552 & -2.974 & -2.678 \\
5.7 & -3.565 & -2.987 & -2.689 & 6.7 & -3.550 & -2.971 & -2.674 \\
5.8 & -3.562 & -2.986 & -2.687 & 6.8 & -3.549 & -2.971 & -2.672 \\
5.9 & -3.558 & -2.975 & -2.679 & 6.9 & -3.546 & -2.971 & -2.672 \\
\hline
\end{tabular}

Note: the critical values are obtained by using 100,000 Monte Carlo trials and the $\mathrm{T}=2000$. 
Table A2. Critical values for the one step intercept and trend case.

\begin{tabular}{cccccccc}
\hline $\mathbf{k}=\mathbf{1}$ & $\mathbf{\% 1}$ & $\mathbf{\%} \mathbf{5}$ & $\mathbf{\% 1 0}$ & $\mathbf{k}=\mathbf{2}$ & $\mathbf{\% 1}$ & $\mathbf{\% 5}$ & $\mathbf{\% 1 0}$ \\
\hline 1.1 & -4.442 & -3.872 & -3.572 & 2.1 & -4.255 & -3.655 & -3.346 \\
1.2 & -4.439 & -3.867 & -3.568 & 2.2 & -4.234 & -3.640 & -3.335 \\
1.3 & -4.433 & -3.865 & -3.564 & 2.3 & -4.215 & -3.612 & -3.303 \\
1.4 & -4.411 & -3.845 & -3.548 & 2.4 & -4.213 & -3.608 & -3.295 \\
1.5 & -4.403 & -3.833 & -3.522 & 2.5 & -4.212 & -3.600 & -3.290 \\
1.6 & -4.389 & -3.812 & -3.501 & 2.6 & -4.201 & -3.600 & -3.285 \\
1.7 & -4.365 & -3.774 & -3.465 & 2.7 & -4.192 & -3.590 & -3.274 \\
1.8 & -4.324 & -3.742 & -3.432 & 2.8 & -4.164 & -3.565 & -3.259 \\
1.9 & -4.294 & -3.717 & -3.402 & 2.9 & -4.154 & -3.565 & -3.253 \\
\hline $\mathrm{k}=3$ & & & & $\mathrm{k}=4$ & & & \\
\hline 3.1 & -4.136 & -3.530 & -3.226 & 4.1 & -4.065 & -3.469 & -3.160 \\
3.2 & -4.126 & -3.523 & -3.220 & 4.2 & -4.058 & -3.468 & -3.153 \\
3.3 & -4.112 & -3.523 & -3.209 & 4.3 & -4.054 & -3.460 & -3.147 \\
3.4 & -4.111 & -3.520 & -3.204 & 4.4 & -4.054 & -3.459 & -3.146 \\
3.5 & -4.100 & -3.506 & -3.191 & 4.5 & -4.047 & -3.458 & -3.143 \\
3.6 & -4.095 & -3.494 & -3.188 & 4.6 & -4.039 & -3.448 & -3.139 \\
3.7 & -4.093 & -3.494 & -3.180 & 4.7 & -4.033 & -3.443 & -3.136 \\
3.8 & -4.092 & -3.491 & -3.180 & 4.8 & -4.029 & -3.433 & -3.132 \\
3.9 & -4.069 & -3.476 & -3.168 & 4.9 & -4.015 & -3.429 & -3.124 \\
\hline $\mathrm{k}=5$ & & & & $\mathrm{k}=6$ & & & \\
\hline 5.1 & -4.032 & -3.431 & -3.122 & 6.1 & -3.992 & -3.403 & -3.093 \\
5.2 & -4.020 & -3.417 & -3.112 & 6.2 & -3.986 & -3.398 & -3.093 \\
5.3 & -4.009 & -3.417 & -3.111 & 6.3 & -3.984 & -3.397 & -3.091 \\
5.4 & -4.009 & -3.416 & -3.111 & 6.4 & -3.977 & -3.392 & -3.090 \\
5.5 & -4.006 & -3.416 & -3.107 & 6.5 & -3.977 & -3.391 & -3.090 \\
5.6 & -4.004 & -3.415 & -3.107 & 6.6 & -3.974 & -3.391 & -3.086 \\
5.7 & -3.995 & -3.411 & -3.101 & 6.7 & -3.973 & -3.390 & -3.086 \\
5.8 & -3.982 & -3.406 & -3.099 & 6.8 & -3.972 & -3.387 & -3.080 \\
5.9 & -3.980 & -3.403 & -3.097 & 6.9 & -3.954 & -3.374 & -3.077 \\
\hline $\mathrm{N}$ & & & & & &
\end{tabular}

Note: The critical values are obtained by using 100,000 Monte Carlo trials and the T $=2000$.

\section{References}

1. He, H.; Modest, D.M. Market Frictions and Consumption-Based Asset Pricing. J. Polit. Econ. 1995, 103, 94-117. [CrossRef]

2. Dumas, B. Dynamic Equilibrium and the Real Exchange Rate in a Spatially Separated World. Rev. Financ. Stud. 1992, 5, 153-180. [CrossRef]

3. Obstfeld, M.; Taylor, A.M. Nonlinear Aspects of Goods-Market Arbitrage and Adjustment: Heckscher's Commodity Points Revisited. J. Jpn. Int. Econ. 1997, 11, 441-479. [CrossRef]

4. Shleifer, A. Inefficient Markets: An Introduction to Behavioural Finance; OUP: Oxford, UK, 2000; ISBN 978-0-19-160689-2.

5. Coakley, J.; Fuertes, A.-M. Nonparametric Cointegration Analysis of Real Exchange Rates. Appl. Financ. Econ. 2001, 11, 1-8. [CrossRef]

6. Hong, H.; Stein, J.C. A Unified Theory of Underreaction, Momentum Trading, and Overreaction in Asset Markets. J. Finance 1999, 54, 2143-2184. [CrossRef]

7. Brock, W.A.; LeBaron, B.D. A Dynamic Structural Model for Stock Return Volatility and Trading Volume. Rev. Econ. Stat. 1996, 78, 94-110. [CrossRef]

8. Brock, W.A.; Hommes, C.H. Heterogeneous Beliefs and Routes to Chaos in a Simple Asset Pricing Model. J. Econ. Dyn. Control 1998, 22, 1235-1274. [CrossRef]

9. Peters, E.E. Fractal Market Analysis: Applying Chaos Theory to Investment and Economics, 1st ed.; Wiley: New York, NY, USA, 1994; ISBN 978-0-471-58524-4.

10. Lux, T. Herd Behaviour, Bubbles and Crashes. Econ. J. 1995, 105, 881-896. [CrossRef]

11. Kapetanios, G.; Shin, Y.; Snell, A. Testing for a Unit Root in the Nonlinear STAR Framework. J. Econom. 2003, 112, 359-379. [CrossRef]

12. Omay, T.; Öznur Kan, E. Re-Examining the Threshold Effects in the Inflation-Growth Nexus with Cross-Sectionally Dependent Non-Linear Panel: Evidence from Six Industrialized Economies. Econ. Model. 2010, 27, 996-1005. [CrossRef]

13. Eruygur, A.Ã.; Omay, T. Terrorism and the Stock Market: A Case Study for Turkey Using STR Models. J. Rev. Glob. Econ. 2014, 3, 220-227. [CrossRef] 
14. Batrancea, I.; Batrancea, L.; Nichita, A.; Gaban, L.; Masca, E.; Morar, I.-D.; Fatacean, G.; Moscviciov, A. An Econometric Approach on Production, Costs and Profit in Romanian Coal Mining Enterprises. Econ. Res.-Ekon. Istraž. 2019, 32, 1019-1036. [CrossRef]

15. Christopoulos, D.K.; León-Ledesma, M.A. Smooth Breaks and Non-Linear Mean Reversion: Post-Bretton Woods Real Exchange Rates. J. Int. Money Financ. 2010, 29, 1076-1093. [CrossRef]

16. Emirmahmutoglu, F.; Omay, T.; Shahzad, S.J.H.; Nor, S.M. Smooth Break Detection and De-Trending in Unit Root Testing. Mathematics 2021, 9, 371. [CrossRef]

17. Enders, W.; Lee, J. A Unit Root Test Using a Fourier Series to Approximate Smooth Breaks*. Oxf. Bull. Econ. Stat. 2012, 74, 574-599. [CrossRef]

18. Enders, W.; Lee, J. The Flexible Fourier Form and Dickey-Fuller Type Unit Root Tests. Econ. Lett. 2012, 117, 196-199. [CrossRef]

19. Omay, T. Fractional Frequency Flexible Fourier Form to Approximate Smooth Breaks in Unit Root Testing. Econ. Lett. 2015, 134, 123-126. [CrossRef]

20. Sercu, P.; Uppal, R.; Van Hulle, C. The Exchange Rate in the Presence of Transaction Costs: Implications for Tests of Purchasing Power Parity. J. Financ. 1995, 50, 1309-1319. [CrossRef]

21. Michael, P.; Nobay, A.R.; Peel, D.A. Transactions Costs and Nonlinear Adjustment in Real Exchange Rates; An Empirical Investigation. J. Polit. Econ. 1997, 105, 862-879. [CrossRef]

22. Davies, R.B. Hypothesis Testing When a Nuisance Parameter Is Present Only Under the Alternatives. Biometrika 1987, 74, 33-43. [CrossRef]

23. Luukkonen, R.; Saikkonen, P.; Teräsvirta, T. Testing Linearity Against Smooth Transition Autoregressive Models. Biometrika 1988, 75, 491-499. [CrossRef]

24. Hamilton, J.D. Time Series Analysis; Princeton University Press: Princeton, NJ, USA, 1994; ISBN 978-0-691-04289-3.

25. Cai, Y.; Omay, T. Using Double Frequency in Fourier Dickey-Fuller Unit Root Test. Comput. Econ. 2021. [CrossRef]

26. Omay, T.; Baleanu, D. Fractional Unit-Root Tests Allowing for a Fractional Frequency Flexible Fourier Form Trend: Predictability of Covid-19. Adv. Differ. Equ. 2021, 2021, 167. [CrossRef] [PubMed] 